\title{
Bacterial Genomic Epidemiology with Mixed Samples
}

\section{Mäklin, Tommi}

2021-11-15

Mäklin , T , Kallonen , T, Alanko , J , Samuelsen , $\varnothing$, Hegstad , K, Mäkinen , V , Corander , J , Heinz , E \& Honkela , A 2021, ' Bacterial Genomic Epidemiology with Mixed Samples ' , Microbial Genomics , vol. 7 , no. 11 , 000691 . https://doi.org/10.1099/mgen.0.000691

http://hdl.handle.net/10138/336390

https://doi.org/10.1099/mgen.0.000691

cc_by

publishedVersion

Downloaded from Helda, University of Helsinki institutional repository.

This is an electronic reprint of the original article.

This reprint may differ from the original in pagination and typographic detail.

Please cite the original version. 


\title{
Bacterial genomic epidemiology with mixed samples
}

\author{
Tommi Mäklin 1,*, Teemu Kallonen²,3, Jarno Alanko ${ }^{4}$, Ørjan Samuelsen ${ }^{5,6}$, Kristin Hegstad ${ }^{5,7}$, Veli Mäkinen ${ }^{4}$, \\ Jukka Corander ${ }^{1,2,3}$, Eva Heinz $z^{2,8} \dagger$ and Antti Honkela ${ }^{4, *}, \dagger$
}

\begin{abstract}
Genomic epidemiology is a tool for tracing transmission of pathogens based on whole-genome sequencing. We introduce the mGEMS pipeline for genomic epidemiology with plate sweeps representing mixed samples of a target pathogen, opening the possibility to sequence all colonies on selective plates with a single DNA extraction and sequencing step. The pipeline includes the novel mGEMS read binner for probabilistic assignments of sequencing reads, and the scalable pseudoaligner Themisto. We demonstrate the effectiveness of our approach using closely related samples in a nosocomial setting, obtaining results that are comparable to those based on single-colony picks. Our results lend firm support to more widespread consideration of genomic epidemiology with mixed infection samples.
\end{abstract}

\section{DATA SUMMARY}

Supplementary figures, tables, and a file describing the implementation details of Themisto have been submitted to the Microbial Genomics figshare account: https://doi.org/106084/ m9figshare15177591v1 [1]. Source code and precompiled binaries (generic Linux and macOS) for both mGEMS and Themisto are freely available in GitHub at https://github.com/ PROBIC/mGEMS (MIT license) and at https://github.com/ algbio/themisto (GPLv2.0 license). A tutorial describing how to reproduce the synthetic mixed samples, bin the mixed reads, and infer the phylogenies is available in the mGEMS GitHub repository. Accession numbers and other information about the synthetic mixed samples is available in Table S1 (available in the online version of this article). The reference data used is available from Zenodo (E. coli doi: 10.5281/zenodo.3724111, E. faecalis doi: 10.5281/zenodo.3724101, S. aureus doi: 10.5281/ zenodo.3724135), and the accession numbers are available in Table S2. Accession numbers for the data used in the in vitro mixture experiments is available in Table S3.

\section{INTRODUCTION}

Public health epidemiology for bacterial infections has been transformed by the use of high-throughput sequencing data to analyse and identify the source of an outbreak and to trace circulating pathogenic strains based on routine surveillance [2-6]. Standard genome-based epidemiological linking of cases requires accurate genome sequences for the pathogens derived from high coverage sequencing data for pure-colony isolates. The isolates are obtained by an enrichment and separation step in the form of a plate culture and subsequent colony picks based e.g. on morphology and colour. Typical workflow of genomic epidemiology may thus necessitate multiple colony picks per sample with the corresponding DNA library preparation and sequencing steps done individually for each of them. DNA isolation, library prep and sequencing require a significant amount of laboratory effort and time per colony, and lead to increased costs since the price of library preparation is becoming comparable to the cost of sequencing itself [7]. This can act

Received 10 June 2021; Accepted 13 September 2021; Published 15 November 2021

Author affiliations: 'Helsinki Institute for Information Technology HIIT, Department of Mathematics and Statistics, University of Helsinki, Helsinki, Finland; ${ }^{2}$ Department of Biostatistics, University of Oslo, Oslo, Norway; ${ }^{3}$ Wellcome Sanger Institute, Hinxton, Cambridgeshire, UK; ${ }^{4}$ Helsinki Institute for Information Technology HIIT, Department of Computer Science, University of Helsinki, Helsinki, Finland; ${ }^{5}$ Norwegian National Advisory Unit on Detection of Antimicrobial Resistance, Department of Microbiology and Infection Control, University Hospital of North Norway, Troms $\varnothing$, Norway; ${ }^{6}$ Department of Pharmacy, UT The Arctic University of Norway, Troms $\varnothing$, Norway; ${ }^{7}$ Research group for Host-Microbe Interactions, Department of Medical Biology, Faculty of Health Sciences, UT The Arctic University of Norway, Tromsø, Norway; ${ }^{8}$ Liverpool School of Tropical Medicine, Liverpool, UK.

${ }^{*}$ Correspondence: Tommi Mäklin, tommi.maklin@helsinki.fi; Antti Honkela, antti.honkela@helsinki.fi

Keywords: genomic epidemiology; pathogen surveillance; plate sweeps; probabilistic modelling; pseudoalignment.

Abbreviations: BGM, Bayesian Gaussian mixture; BIGSdb, bacterial isolate genome sequence database; bp, base pair; ENA, European Nucleotide

Archive; GTR, generalized time reversible; MLST, multilocus sequence type; MRSA, methicillin-resistant Staphylococcus aureus; NCBI, National

Center for Biotechnology Information; RAM, random-access memory; ST, sequence type.

†These authors contributed equally to this work

Data statement: All supporting data, code and protocols have been provided within the article or through supplementary data files. One supplementary table and seven supplementary figures are available with the online version of this article.

000691 ( 2021 The Authors 
as a barrier to more widespread genomic pathogen surveillance even in well-resourced public health laboratories, and prevent application of genomic epidemiology altogether in poorer settings.

Whole-genome shotgun metagenomics has been proposed as a solution for getting rid of the culturing step entirely when storing the isolates for record keeping or later phenotyping is not necessary. In this approach, sequencing is performed directly on the DNA extracted from the original sample and the resulting reads computationally binned or assembled. While tools capable of pangenome-based analyses [8], metagenome assembly [9-12], or taxonomic binning [13-15] from metagenomic short-read sequencing data have been developed, these methods typically require that the samples do not contain many closely related organisms. In particular, the strain-variation within a species is assumed to be large enough not to be confused with sequencing errors or variation in the assembly graph [16]. When more complex strain-level diversity is present, benchmarking these tools shows reduced performance in both taxonomic binning and metagenomic assembly [17-20]. In practice, natural strain-level variation is harboured ubiquitously in epidemiologically relevant samples [21-30] and it is reflected by the transmission events occurring between individuals and their environment [31]. Although some sample types may be dominated by one or two strains [32], direct sequencing of clinical samples may result in an overabundance of host DNA [29, 33-35], or lack detection power for strains with low abundance in environments with high species diversity $[19,33,36]$. These challenges are overcome in genomic epidemiology by enriching the target species through the use of plate cultures. Since established protocols and growth media are available for most bacteria of clinical relevance [37], enrichment provides an effective means to deplete the host DNA and increase the sequencing depth for target organisms when working with well-characterized species.

In this article, we introduce the mGEMS pipeline for performing genomic epidemiology with mixed cultures from samples that may harbour multiple closely related bacterial lineages. mGEMS requires only a single culturing and library preparation step per sample, which can significantly reduce the cost of performing genomic epidemiology in the standard public health setting and make the whole process more streamlined. We demonstrate the effectiveness of our approach in SNP calling and phylogenetic analyses by using in vitro mixed samples of Escherichia coli and Enterococcus faecalis strains, as well as DNA reads synthetically mixed from closely related samples obtained from previous genomic epidemiology studies $[30,38,39]$ tracking E. faecalis, E. coli and Staphylococcus aureus in public health settings. Likewise, the E. coli and E. faecalis strains used in the in vitro samples were hospital isolates and selected as representatives of clinically highly relevant sequence types. Our results illustrate that accurate transmission and case-linking analyses are possible at reduced cost levels by enabling sample de-mixing and subsequent variant calling.

\section{Impact Statement}

The reduced cost of high-throughput sequencing has enabled genomic epidemiology to be adopted widely for public health applications worldwide. In this paper we propose the mGEMS pipeline that promises to make genomic epidemiology even more affordable by replacing separate analysis of colony picks with a single analysis per plate sweep, which significantly reduces the costs of the DNA isolation, library preparation and sequencing steps, facilitating scaling up genomic epidemiology studies. Our mGEMS pipeline provides a tool to perform typical genomic epidemiological analyses directly from plate sweeps of all colonies on a single plate with a single DNA extraction, library preparation and sequencing step. We expect mGEMS to facilitate more widespread application of genomic epidemiology in public health laboratories and enable the development of entirely novel types of analyses and pipelines.

Key parts of our pipeline presented in this paper are the mGEMS binner for short-read sequencing data, and the scalable pseudoaligner Themisto, which provides an exact version of the kallisto pseudoalignment algorithm [40] and significantly reduced RAM usage for large reference databases of single-clone sequenced bacterial pathogens. Together with recent advances in both probabilistic modelling of mixed bacterial samples [41] and genome assembly techniques [42], these methods form the mGEMS pipeline. A central step in mGEMS is an application of the recent mSWEEP method [41], which estimates the relative abundance of reference bacterial lineages in mixed samples using pseudoalignment and Bayesian mixture modelling. While Themisto enables upscaling of mSWEEP to significantly larger reference databases, the mGEMS binner is a novel sequencing read binning approach. Our binner is based on leveraging probabilistic sequencing read classifications to reference lineages from $\mathrm{mSWEEP}$, and notably allowing a single read to be assigned to multiple bins. Using mGEMS to bin the reads in the original mixed samples produces sets of reads closely resembling standard isolate sequencing data and additionally acts as a denoising step for removing possible contaminant DNA. These advances enable efficient application of the existing tools for genomic epidemiology to the analysis of mixed culture samples, paving the way to a more widespread consideration of genomic epidemiology for public health applications.

\section{METHODS mGEMS workflow}

Our pipeline for performing genomic epidemiology with short-read sequencing data from mixed samples, mGEMS, requires as input the sequencing reads and a reference database representing the clonal variation in the organisms 
likely contained in these reads. The reference database must additionally be grouped accordingly into clonal groups representing lineages within the species. We used either the multilocus sequence types (E. faecalis experiments) or sublineages within the sequence types (E. coli and S. aureus experiments) as the clonal grouping. With these pre-processing steps performed, the first step in the mGEMS pipeline is to pseudoalign [40] the sequencing reads against the reference database using our scalable implementation of (exact) pseudoalignment with the Themisto software (in this article we used v0.1.1 with the optional setting to also align the reverse complement of the reads enabled). The pseudoalignments and the clonal grouping are then supplied as input to the mSWEEP software (v1.3.2; doi: 10.5281/zenodo.3631062 [41], with default settings) which estimates the relative sequence abundances of the clonal groups in the mixed sample. Consequently, mSWEEP produces a probabilistic assignment of the sequencing reads to the different reference clonal groups. This tentative assignment is subsequently processed by the mGEMS binner (v0.1.1, default settings), which assigns the sequencing reads to bins that correspond to a single reference clonal group - with a possibility for a sequencing read to belong to multiple bins. As the last step, the bins are (optionally) assembled with the Shovill (v0.9.0, with default settings [42]) assembly pipeline. mGEMS and Themisto are freely available on GitHub (https://github.com/ PROBIC/mGEMS and https://github.com/algbio/themisto).

\section{Reference data for mSWEEP and mGEMS}

We used assemblies of three different sets of sequencing data as the reference for the three different experiments presented. The three different reference datasets represent a local (S. aureus experiment, [30]), a national (E. coli, [43]) and a global collection (E. faecalis downloaded from the NCBI) of strains from these species. Accession numbers and multilocus sequence types for the reference data are available in Supplementary Table 2 accompanied with rudimentary assembly statistics from both the isolate sequencing data and the assemblies from the mGEMS pipeline. In each experiment, we only aligned against the reference sequences from the relevant species.

In the E. coli experiments, our collection of 218 E. coli ST131 isolates originated from the British Society for Antimicrobial Chemotherapy's bacteraemia resistance surveillance programme and were originally isolated from 11 hospitals across England [43]. These isolates were assigned to six ST131 sublineages (A, B0, B, C0, C1, or C2) as described in a previous study [43]. As the reference sequence for calling the SNPs in building the phylogeny, we used the ST131 strain NCTC13441 (European Nucleotide Archive [ENA] sequence set UFZF01000000).

For the E. coli experiment where the six sublineages were further split, the split was generated by clustering the sequences with PopPUNK (v2.3.0, [44]) with the BGM model using four components and then performing the subsequent refinement step. The resulting PopPUNK clustering was combined with the sublineage numbering by concatenating the two together. This clustering is included in Supplementary Table 2.

The global collection of $E$. faecalis reference data was obtained by downloading all available E. faecalis assemblies (1484 as of 2 February 2020) from the NCBI, which were assigned to STs with the BIGSdb $[45,46]$ mlst software at pubmlst. org and assigned to the assemblies with the mlst software (v2.18.1) [47]. Sequence type could not be determined for 177 assemblies. These were discarded, leaving a total of 1307 assemblies assigned to 203 distinct sequence types. We used the ST6 strain V583 [48] as the reference for SNP calling (NCBI RefSeq sequences NC_004668.1-NC_004671.1).

The $S$. aureus reference data were obtained from the same study as the experiment data [30]. We used Shovill (v0.9.0 [42], with default settings) to assemble the isolate sequencing reads from the first sampling of the staff members at the veterinary hospital, and assigned the assembled sequences to the ST22 sublineages according to the information provided in original study [30]. The reference sequence used in calling the SNPs was the ST22 strain HO 50960412 ([49], ENA sequence HE681097.1)

If the reference sequence in any of the experiments consisted of multiple contigs, we concatenated the contigs together by adding a 100-base gap between them. The final reference file that was used as input for Themisto indexing was produced by concatenating all reference sequences processed in this way together.

\section{In vitro mixture experiment setup}

We first generated reference genomes for the three E. coli and three $E$. faecalis strains used in the in vitro benchmarking of mGEMS. In order to obtain as accurate reference genomes as possible, we combined short-read Illumina sequencing data (Table S3) with long-read Oxford Nanopore sequencing data.

In the first mixture experiment, single colonies of each strain were grown up overnight in liquid medium (LB broth [SigmaAldrich] for E. coli and brain heart infusion broth [Fluka Analytical] for E. faecalis), and DNA extracted for short-read sequencing. The DNA concentration was quantified using the Qubit system (Invitrogen) and purified DNA, diluted to 30 $\mathrm{ng} \mathrm{\mu l}^{-1}$, from the three strains of $E$. coli and the three strains of $E$. faecalis were used to prepare three different mixtures with varying ratios (1:1:1, 1.4:1.4:0.2, and 2.2:0.4:0.4; Table 1). These mixtures were then prepared for Illumina sequencing, and analysed as described earlier in the manuscript. All sequencing data generated for these mixture experiments is available under BioProject PRJNA720284.

For the second experimental setup, the six reference strains were again grown in single-clone cultures overnight as described above, and 1:1:1 mixtures of the liquid cultures from the three strains per species were made, centrifuged, and used as sample for DNA extraction for short-read sequencing. Unlike in the first experimental setup, in this setup the concentrations of the different strains were not measured 
Table 1. Mixture concentration ratios in the six in vitro experiment samples. The column labels contain the sample identifiers and lineages for the strains that were mixed in each of the six experiments, represented by the rows of the table. The values are the proportions of each strain in the sample out of the total concentration of $30 \mu \mathrm{l}$.

\begin{tabular}{|c|c|c|c|}
\hline & $\begin{array}{c}\text { NORM7910041 } \\
\text { E. coli ST131-C2-4 }\end{array}$ & $\begin{array}{c}\text { NORM7911464 } \\
\text { E. coli ST131-C2-6 }\end{array}$ & $\begin{array}{c}\text { NORM7908673 } \\
\text { E. coli ST131-A-14 }\end{array}$ \\
\hline $\begin{array}{l}\text { Exp. } 1 \text { E. coli } \\
(1: 1: 1)\end{array}$ & 0.33 & 0.33 & 0.33 \\
\hline $\begin{array}{l}\text { Exp. } 2 \text { E. coli } \\
(1.4: 1.4: 0.2)\end{array}$ & 0.47 & 0.47 & 0.07 \\
\hline $\begin{array}{l}\text { Exp. } 3 \text { E. coli } \\
(2.2: 0.4: 0.4)\end{array}$ & $\begin{array}{c}51271926 \\
\text { E. faecalis ST6 }\end{array}$ & $\begin{array}{c}51271052 \\
\text { E. faecalis ST40 }\end{array}$ & $\begin{array}{c}51271223 \\
\text { E. faecalis ST28 }\end{array}$ \\
\hline $\begin{array}{l}\text { Exp. } 4 \text { E. faecalis } \\
(1: 1: 1)\end{array}$ & 0.33 & 0.33 & 0.33 \\
\hline
\end{tabular}

with Qubit and thus are not available beyond the initial $1: 1$ : 1 mixture of the liquid cultures.

\section{DNA extraction}

DNA was extracted using the MagAttract HMW kit (Qiagen) for Oxford Nanopore sequencing, and the DNeasy Blood and Tissue kit (Qiagen) for short-read sequencing.

\section{Sequencing}

DNA libraries for long-read sequencing were prepared using the Ligation Sequencing Kit SQK-LSK109 (Oxford Nanopore) in combination with the native barcoding kits EXP-NBD104 and EXP-NBD114 (both Oxford Nanopore) according to the manufacturer's instruction. DNA was sequenced using an Oxford Nanopore GridION on a R9.4.1 flow cell with an input of $337.5 \mathrm{ng}$. For short-read sequencing, DNA libraries were prepared using the Nextera XT DNA library kit (Illumina) and sequenced on an Illumina NextSeq550 using a midoutput flow cell, 300 cycles and $2 \times 150$ bp paired-end set-up.

\section{Reference genome assembly for the in vitro mixed experiments}

We performed hybrid assembly from the long and short reads by first assembling only the long-reads and then using the short-reads for error correction. The initial long-read only assembly was created from the raw long-read sequencing data with Flye (v2.8.2, [50]) and polished with quality controlled long reads (QC'd with filtlong v0.2.0; https://github.com/ rrwick/Filtlong) using medaka (v1.2.1, https:/github.com/ nanoporetech/medaka/). The short reads were used for error correction by first quality controlling them with fastp (v0.21.0, [51]) and then using pilon (v1.23, [52]) to perform the error correction on the long-read assembly. This procedure resulted in closed chromosome and plasmid sequences for all six reference strains. The short reads, long reads, and the produced genomes have been submitted and made available in standard repositories (Table S3).

\section{Synthetic mixture generation}

We produced our three synthetic mixture sets by synthetically mixing together the isolate sequencing data from distinct lineages in each of the three studies [30, 38, 39]. In the E. coli experiments, we produced ten mixed samples with one strain from each of the three main ST131 lineages (A, B, or C) in each sample. In the E. faecalis experiments, we mixed together seven strains from seven different sequence types to produce a total of 12 mixed samples. The strains included in each sample were chosen at random without replacement in the E. coli and $E$. faecalis experiments. The $S$. aureus mixed samples were produced by randomly mixing together one strain from each of the three sublineages with replacement while ensuring that each strain appears at least once. The sequencing data that were used in the reference dataset were not included in any of the experiments. In all three experiment sets, we used all available sequencing data in the mixed samples, resulting in 8-15 million reads in the experiments. Table S1 contains the accession numbers and lineage assignments of the isolate sequencing data in each sample, as well as the assembly statistics from both isolate sequencing and the synthetic mixed samples processed with mGEMS.

\section{Pseudoalignment}

We used Themisto (v0.1.1) with the default settings. Themisto is a $k$-mer-based pseudoalignment tool which encodes sets of $k$-mers as a succinct coloured de Bruijn graph. A read is considered to pseudoalign against a reference sequence if at least one $k$-mer of the read is found in the reference, and each $k$-mer of the read is either found in the reference or 
not found at all in the database of all references. This can be seen as an exact version of the pseudoalignment algorithm implemented by the tool kallisto [40]. Unlike kallisto, Themisto constructs the alignment index utilizing external memory, leading to massively reduced RAM consumption during index construction. Additionally, the index structure in Themisto is implemented using advanced compact data structures [53] to minimize the amount of memory required to store the index. Themisto is also able to exploit redundancy in the colour sets of the nodes to compress the index further.

In all of our experiments, the index was constructed using 31-mers. Themisto does not distinguish between paired-end reads and single reads, so we decided to consider a paired-end read as pseudoaligned only when both fragments pseudoaligned. We have included this functionality for supporting paired-end reads in both the MSWEEP and MGEMS software implementations.

\section{Abundance estimation and probabilistic read assignment}

We used the mSWEEP [41] software (v1.3.2; doi: 10.5281/ zenodo.363106210.5281/zenodo.3631062) with default settings. The programme was altered to support pseudoalignments from Themisto, and to output the read-level probabilistic assignments to the reference lineages. We also improved the scalability of mSWEEP by parallelizing the abundance estimation part and reducing memory consumption. These alterations have been included in versions v1.3.2 (Themisto and mGEMS support) and v1.4.0 (parallelization and memory usage improvements) of the software.

\section{Read binning}

In order to collect all reads in a mixed sample that likely originate from the same target lineage, we consider a binning strategy that allows associating the same read with multiple reference lineages. We assume that each reference lineage is represented by, at most, only one target sequence in the mixed sample, and that the sets of reference sequences capture the variation in the reference lineages sufficiently to use them as a substitute for the target sequence which may not be included in the reference sequences. In our formal treatment of the task of binning a set of sequencing reads, we define the task in terms of finding $K$ subsets (bins), one for each reference lineage $k=1, \ldots, K$, of the full sets of reads $R=\left\{r_{1}, \ldots, r_{N}\right\}$ denoted by $G_{k} \subset R$ that contain reads likely originating from the target sequence belonging to the reference lineage $k$. The reads assigned to each subset $G_{k}$ are determined based on read-level probabilities $\gamma_{n, k}, \sum_{k=1}^{K} \gamma_{n, k}=1, n=1, \ldots, N$ to assign the read $r_{n}$ into the reference lineage $k$ by defining the subsets $G_{k}$ such that

Equation 1:

$$
G_{k}=\left\{r_{n}: \gamma_{n, k} \geq q_{k}\right\}
$$

holds for some threshold $q_{k} \in[0,1]$ which may vary between the lineages $k$. The formulation in Equation (1) has the benefit of allowing the read $r_{n}$ to possibly belong to several subsets
$G_{k}$, which is an important property for dealing with multiple closely related lineages in the same mixed sample.

In order to find a suitable value for the threshold $q_{k}$, and to determine the corresponding assignment rule, we consider two binary events: (1) $I_{n, k}$ : the reference lineage $k$ generated the read $r_{n}$, and (2) $J_{n, k}$ : the true nucleotide sequence represented by the read $r_{n}$ is part of the target sequence belonging to the reference lineage $k$. Knowing the probability of the event $J_{n, k}$ would directly enable us to assess the plausibility of assigning the read $r_{n}$ to the reference lineage $k$ but its value is difficult to estimate directly. However, we can determine and write down the values of the conditional probabilities $P\left[I_{n, k}=1 \mid J_{n, k}=0\right]$ and $P\left[I_{n, k}=1 \mid J_{n, k}=1\right]$ as

Equation 2:

$$
\begin{gathered}
P\left[I_{n, k}=1 \mid J_{n, k}=0\right]=0, \text { and } \\
P\left[I_{n, k}=1 \mid J_{n, k}=1\right]=\frac{\theta_{k}}{\sum_{c: I_{n, c}=1} \theta_{c}}
\end{gathered}
$$

where $\theta_{k}$ is the proportion of reads from the reference lineage $k$ , and $\sum_{c: I_{n, c}=1} \theta_{c}$ is the proportion of reads from any reference lineages $\left\{c: J_{n, c}=1\right\}$ which contain the sequence represented by the read $r_{n}$. The conditional probabilities in Equation (2) allow us to write the unconditional probability $P\left[I_{n, k}=1\right]$ as

Equation 3:

$$
\begin{aligned}
& P\left[I_{n, k}=1\right]=P\left[I_{n, k}=1 \mid J_{n, k}=0\right] P\left[I_{n, k}=0\right]+ \\
& P\left[I_{n, k}=1 \mid J_{n, k}=1\right] P\left[J_{n, k}=1\right] \\
& \Leftrightarrow P\left[I_{n, k}=1\right]=\frac{\theta_{k}}{\sum_{c: I_{n, c}=1} \theta_{c}} P\left[I_{n, k}=1\right] .
\end{aligned}
$$

Using the formulation in Equation (3) and the fact that we can approximate

$\frac{\theta_{k}}{\sum_{c: I_{n, c}=1} \theta_{c}} \approx \theta_{k}$ if we assume that the mixed sample is mostly composed of closely related organisms (the denominator $\sum_{c: I_{n, c}=1} \theta_{c}$ approaches 1), we can rewrite Equation (3) as

Equation 4:

$$
P\left[I_{n, k}=1\right] \approx \theta_{k} P\left[J_{n, k}=1\right]
$$

Equations (4) and (3) together imply that if the value of the probability $P\left[I_{n, k}=1\right]$ that the read $r_{n}$ was generated from the lineage $k$ exceeds the relative abundance $\theta_{k}$ of that lineage in whole sample $\left(P\left[I_{n, k}=1\right] \geq \theta_{k}\right)$, then the value of the probability $P\left[J_{n, k}=1\right]$ that the nucleotide sequence represented by the read $r_{n}$ is contained in the target sequence from the reference lineage $k$ must be 'large' $\left(P\left[J_{n, k}=1\right] \rightarrow 1\right)$. This statement about the magnitude of $P\left[J_{n, k}=1\right]$ derives from our assumption that the denominator in Equation (3) is close to 1.

Since we have an estimate of the probabilities $P\left[I_{n, k}=1\right]$ available in the form of the read-level probabilistic assignments $\gamma_{n, k} \approx P\left[I_{n, k}=1\right]$, we can plug these values in Equation (4) and use the result to derive the assignment rule

Equation 5:

$$
\text { if } \gamma_{n, k} \geq \theta_{k} \text {, assign the read } r_{n} \text { to } G_{k}
$$


The assignment rule in Equation (5) gives us a way to assess the validity of the statement contained in the probability $P\left[J_{n, k}=1\right]$ which we could not estimate directly.

Because of computational accuracy, we cannot obtain meaningful relative abundance estimates $\theta_{k}$ for lineages with a relative abundance less than $\frac{1}{N}$ (less than one read from the lineage $k$ in the sample). Since there are $K$ lineages in total, in the worst-case scenario $K_{N} \frac{1}{N}$ units of the relative abundance fall into this meaningless range. Therefore, only a fraction of the total relative abundance of 1 can be considered to be accurately determined when using computed values of $\theta_{k}$, and this fraction $d$ is determined in the worst-case scenario through the formula

Equation 6:

$$
d=1-K \frac{1}{N}
$$

Equation (6) means that when evaluating the validity of the assignment rule presented in Equation (5) with computed values, we have to replace $\theta_{k}$ with the value $d \theta_{k}$ which depends on the value of $d$ in Equation (6). Merging the result from (Equations $5 ; 6$ ) leads us to the final assignment rule of if

Equation 7:

$$
\text { if } \gamma_{n, k}>d \theta_{k} \text {, assign the read } r_{n} \text { to } G_{k}
$$

In practice, reads which pseudoalign to exactly the same reference sequences have identical values $\gamma_{n, k}$. The reads can thus be assigned to equivalence classes defined by their pseudoalignments, which enables a speedup in the implementation of the binning algorithm by considering each equivalence class as a single read. Due to this speedup and the computational simplicity of evaluating the assignment rule in Equation (7), the memory footprint of the mGEMS binner is determined by the number of equivalence classes and reference lineages in the input pseudoalignment and the runtime limited by disc I/O performance.

\section{Genome assembly from mGEMS bins}

After binning the sequencing reads in our experiments with the aforementioned assignment rule, we assembled the sequencing reads assigned to the bins using the Shovill (v0.9.0 [42], with default settings) assembly optimizer for the SPAdes assembler $[54,55]$. This step concludes what we in this article call the mGEMS pipeline.

\section{SNP calling and phylogeny reconstruction}

We used snippy (v4.4.5, [56]) to produce a core SNP multiplesequence alignment from the assembled contigs. Since the $E$. coli and $S$. aureus strains used were from the same sequence type, the core alignment for these two species contained almost the whole genome. After running snippy, we used snp-sites (v2.5.1, [57]) to remove sites with ambiguous bases or gaps from the alignment (E. coli experiments only) and then ran RAxML-NG (v0.8.1, [58]) to infer the maximumlikelihood phylogeny under the GTR+G4 model. Since some of the $S$. aureus strains from the same clade were identical, we changed the default value of the minimum branch length parameter in RAxML-NG to $10^{-10}$ in the S. aureus experiments and printed the branch length with eight decimal precision to identify branches of length zero. In all experiments, we ran RAxML-NG with 100 random and 100 maximum parsimony starting trees, and performed 1000 bootstrapping iterations to infer bootstrap support values for the branches. We used the phytools R package (v0.6-99, [59]) to perform midpoint rooting for the tree, and the ape R package (v5.3, [60]) to create the visualisations.

\section{RESULTS}

\section{Read binning and genome assembly from mixed samples with mGEMS}

Our mGEMS read binning algorithm, part of the mGEMS pipeline (Fig. 1), requires probabilistic assignments of sequencing reads to reference taxonomic units (lineages or sequences) and an estimate of the relative sequence abundance of these same references in the full set of reads. mGEMS then bins the reads by assigning a read to a bin (corresponding to a target sequence from a given reference lineage) if the read-level assignment probability of the lineage is greater than or equal to the sequence abundance of that particular lineage in the full set of reads. Notably, this algorithm allows a single sequencing read to be assigned to multiple bins which is a crucial feature for considering strain-level variation. As shown in the Methods section, this algorithm assigns reads to reference lineages only if the sequence represented by a read is likely contained in a target sequence that belongs to the reference lineage.

In the pseudoalignment part of the pipeline (Fig. 1), we use our own more efficient and accurate implementation of the pseudoalignment algorithm in kallisto [40], called Themisto, to pseudoalign the sequencing reads against the reference sequences. Themisto is based on using coloured de Bruijn graphs to represent the reference sequences, and utilizes disc storage during indexing to control the amount of RAM required to construct the pseudoalignment index. These choices lead to Themisto aligning a similar number of reads per hour as kallisto, while being 70 times faster to load in an example pseudoalignment index consisting of $3682 \mathrm{E}$. coli sequences $(28 \mathrm{~min}$ for kallisto and $0.55 \mathrm{~min}$ for Themisto; Supplementary Methods). Implementation of the method is described in more detail in Supplementary Methods.

The pseudoalignments from Themisto are used as input to the mSWEEP method [41] to estimate the probabilistic read assignments and whole-sample relative sequence abundances. These values provide the necessary input to the mGEMS binner which assigns the sequencing reads to the bins. Finally, we use the Shovill [42] assembly optimizer for the SPAdes assembler $[54,55]$ to assemble the bins. On an example synthetic mixed sample (the E. coli sample with the most reads), the full mGEMS pipeline took 112 min to run (Themisto $26 \mathrm{~min}$, mSWEEP $4 \mathrm{~min}$, mGEMS 


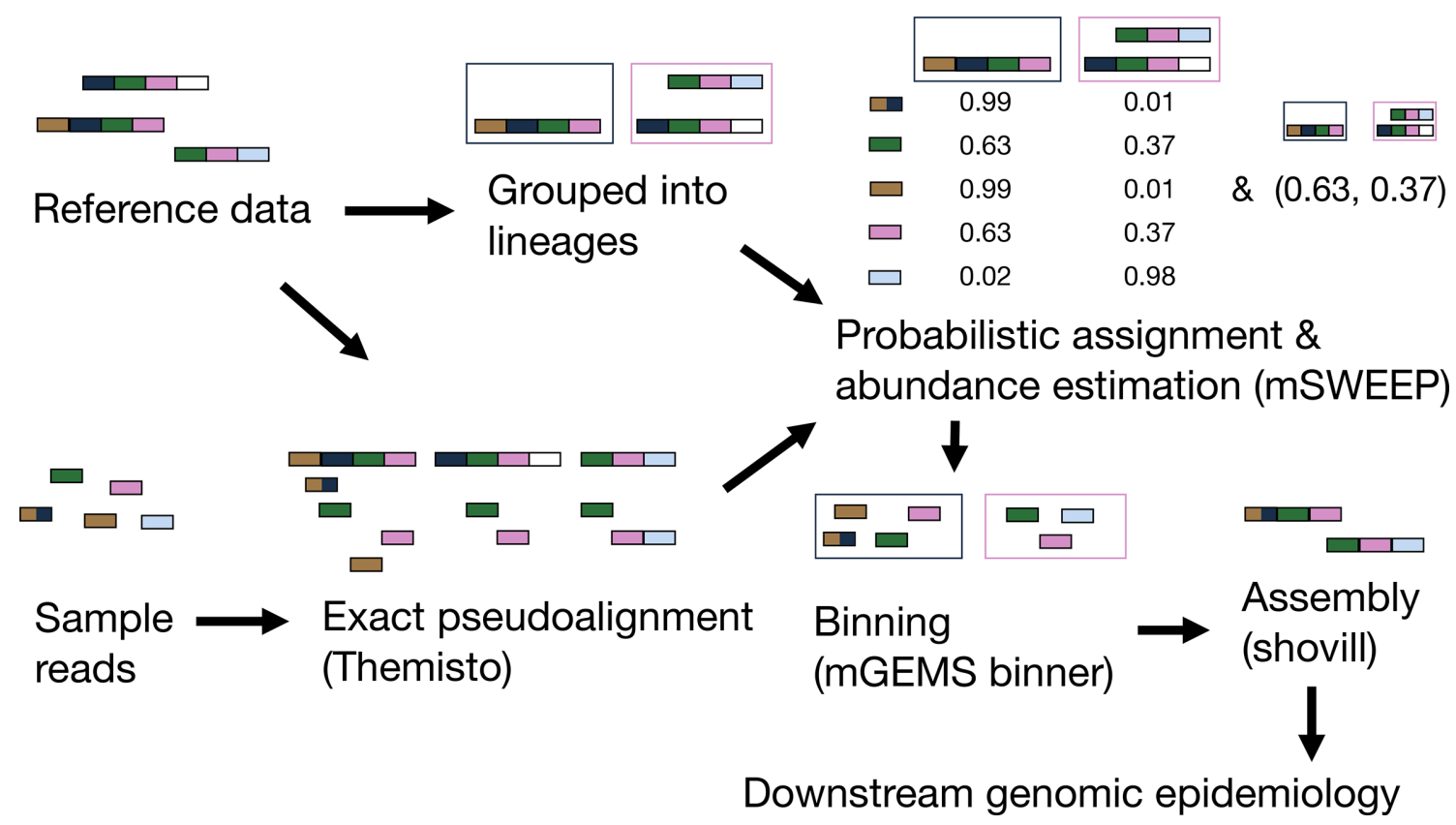

Fig. 1. Flowchart describing a genomic epidemiology workflow with the mGEMS pipeline. The figure shows the various steps of the pipeline. Steps with programme names in brackets constitute the parts of the mGEMS pipeline. Presented values from mSWEEP and mGEMS binner are the actual results of running the pipeline with the described input.

binner $16 \mathrm{~min}$, and Shovill $66 \mathrm{~min}$ ) using two threads on a laptop computer with two processor cores and 16 gigabytes of memory. C++ implementations of both the mGEMS binner and the Themisto pseudoaligner are freely available on GitHub (https://github.com/PROBIC/mGEMS, MIT license, and https://github.com/algbio/themisto, GPLv2 license).

\section{Overview of the experiments used in benchmarking mGEMS}

We assessed the accuracy and effectiveness of mGEMS by considering data from three genomic epidemiological studies [30, 38, 39] and by generating a benchmarking dataset of in vitro mixed samples with measured DNA concentrations. The in vitro dataset was generated by first growing three strains of E. coli and three strains of E. faecalis separately, resulting in six overnight cultures in liquid medium. Next, the amount of DNA extracted from the overnight cultures was measured and six mixtures, each consisting of three strains of either E. coli or E. faecalis, with known concentrations of DNA for each isolate, were created. This resulted in a benchmark dataset where the relative abundances of the different strains in each mixture are known. We also generated two additional mixtures where the E. coli or E. faecalis strains were mixed in $1: 1$ : 1 proportions from the liquid culture without measuring the amount of DNA, and the DNA extraction was then performed on these already-mixed bacterial samples. To our knowledge, these benchmarking samples constitute the first published dataset where DNA from three strains of the same species has been mixed with known concentrations, providing an important resource for development of methods aimed at untangling strain-level variation.

In the synthetic mixture experiments, we used sequencing reads from previously published genomic epidemiological studies [30, 38, 39] as the basis for creating synthetic mixture data. The synthetic mixtures were processed with the mGEMS pipeline, and the output was compared against the benchmark of having non-mixed data available by running the same epidemiological analyses on both the mGEMS output and the non-mixed data. The synthetic experiments presented are: (1) mixing reads from three clones of $E$. coli sequence type (ST) 131 sublineages obtained from a study of multidrug-resistant E. coli ST131 strains circulating in a long-term care facility in the UK [38], (2) mixing reads from seven E. faecalis STs identified in a study of the population structure of hospital-acquired vancomycin-resistant E. faecalis lineages in the UK and Ireland [39], and (3) mixing reads from three $S$. aureus ST22 sublineages from a study of the transmission network of methicillin-resistant $S$. aureus (MRSA) among staff and patients at an UK veterinary hospital [30]. We also provide three different approaches to constructing the reference datasets for the pseudoalignment step: (1) a national (UK) collection of E. coli ST131 isolates associated with bacteremia [43], (2) a global collection of all available $E$. faecalis genome assemblies from the NCBI as of 2 February 2020 , and (3) a local collection of $S$. aureus sequencing 
data from the staff members at the veterinary hospital at the earliest possible time point in the same study [30]. A detailed description of the generated experiments and the accession numbers of the isolate sequencing and reference data used is presented in the Methods section.

\section{Evaluation of mGEMS and mSWEEP on the in vitro benchmark data}

We first evaluated the accuracy of mGEMS and mSWEEP on the six in vitro experimental samples, where the true relative abundances of the three strains in each sample are known. In the E. faecalis samples each of the three strains originated from a different multilocus sequence type (MLST) and the measurements were accordingly performed on the level of the MLST grouping [45]. In the E. coli samples, the strains originated from sublineages within ST131 as defined in a previous study [43], with one strain from sublineage ST131-A and two from sublineage ST131-C2. In order to distinguish between the two strains from ST131-C2, we further split the strains based on their accessory genomes using PopPUNK [44], which provided us with a grouping where all three strains were split into three separate groups (A-14, C2-4, and C2-6), enabling us to differentiate between them with mSWEEP and mGEMS.

We assessed the accuracy of mGEMS by comparing the results of SNP calling from a hybrid long +short read assembly obtained from a single-colony derived sample of the strains used in the in vitro mixed experiments with calling the SNPs from an assembly obtained by processing the mixed experiment samples with the mGEMS pipeline. The results of the SNP calling are highly similar in both datasets (Fig. 2a, b) with the exception of the E. coli ST131C2-6 strain from the experiment labelled 'Exp 2 E. coli'. In this experiment, the sample consisted of equal amounts of DNA from the ST131 C2-4 and C2-6 strains and a small amount of ST131-A-14, causing some confusion between the reads originating from the closely related C2-4 and C2-6 strains which resulted in a difference between the observed and expected SNP counts.

Similarly, the mSWEEP relative abundance estimates for both the E. coli and E. faecalis samples correspond well with the true values when measured by both absolute and relative error (Fig. 2c, d, respectively). Slightly higher errors were observed in the estimates for the E. coli ST131 C2-4 and C2-6 strains when compared to the estimates for the E. coli ST131 A-14 strain and all three E. faecalis strains. Akin to the results of SNP calling with mGEMS, these differences in the relative abundance estimates are likely a result of using the highly detailed E. coli within-ST clustering, which is significantly harder to differentiate than the between-ST clustering used for E. faecalis. Regardless, in all cases, there are no false positive or false negative detections of lineages reported in the MSWEEP relative abundance estimates.

\section{SNPs from synthetic mixtures match SNPs called from isolate data}

In the first synthetic mixture benchmark, we compared the accuracy of SNP calling with the snippy software (v4.4.5) [56] from the bins obtained by processing the abundance estimation results from the mixed samples with the mGEMS binner with the results of the same analyses from the isolate sequencing data (Fig. 3). In the E. coli and E. faecalis experiments (Fig. 3a, b, respectively), the SNPs were called from assembled contigs while in the $S$. aureus experiment (Fig. 3c), we called the SNPs directly from the sequencing reads because calling the SNPs from the contigs resulted in poorer performance (Fig. S1). In all experiments, the SNPs called from the mixed samples closely resemble the results of isolate sequencing data in both the samples that are similar and dissimilar to the reference sample. Although in the E. coli experiment mGEMS produced slightly more SNPs on average, the results were consistently higher for all samples and did not affect the results of the analyses presented further in this article.

We suspected that the observed differences in the SNP counts may have been caused by issues in the sequence assembly due to mGEMS allowing a read to belong to multiple bins, which results in variable coverage between the regions with and without the clade-specific SNPs. We tested this assumption by replacing the Shovill assembler in the mGEMS pipeline with metagenomic assemblers, which naturally handle variable coverage. Using the metagenomic assemblers marginally improved the results in some of the experiments (Figs 3d and S2) with MEGAHIT [10, 11] in particular outperforming Shovill when the coverage is markedly varied like in the S. aureus experiment. However the improvements were not drastic enough to decisively confirm our suspicions about the accuracy of the SNP calling being limited by the choice of assembler. We did observe that when measured by reference-independent assembly statistics (sum of all contig lengths, total number of contigs, sequence length of the shortest contig at 50\% genome length $\mathrm{N} 50$, and the smallest number of contigs whose sum is at least $50 \%$ of the genome length L50), the statistics obtained from the standard configuration of mGEMS with the Shovill assembler resemble those from isolate sequencing data.

We further assessed the accuracy of the called SNPs by fitting a Bayesian linear regression model to the same SNP data with the isolate results as the sole explanatory variable and the results from the bins or the metagenomic assemblers as the response variable (Figs 3 and S2) using the brms R package [61-63]. In both the E. coli ST131 sublineage and the E. faecalis experiments, the $95 \%$ posterior credible interval for the slope from mGEMS with all assembler choices except metaSPAdes contains the correct value of 1.0. The S. aureus experiments produce worse $95 \%$ credible intervals for the slope compared to the E. coli and $E$. faecalis experiments with none of the intervals containing the correct value. However, the regression model is not well 
(a)

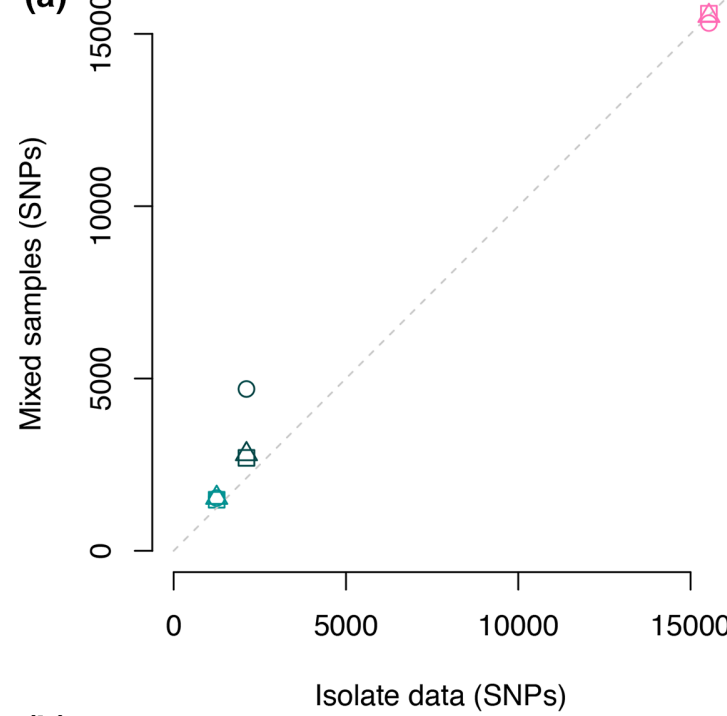

(b)

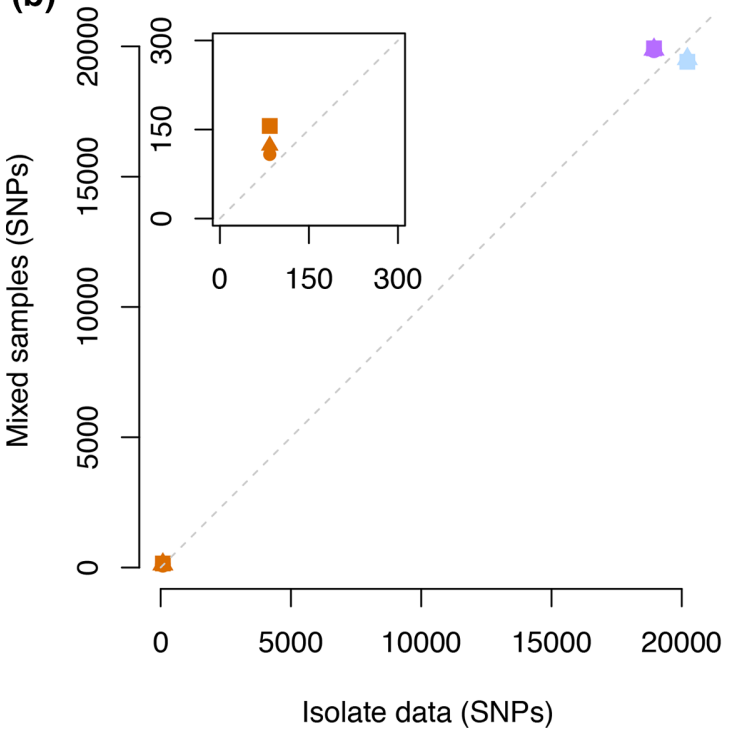

口 Exp. 1 E. coli ST131-C2-4

$\square$ Exp. 1 E. coli ST131-C2-6

$\square$ Exp. 1 E. coli ST131-A-14

- Exp. 2 E. coli ST131-C2-4

- Exp. 2 E. coli ST131-C2-6

- Exp. 2 E. coli ST131-A-14

$\triangle$ Exp. 3 E. coli ST131-C2-4

$\triangle$ Exp. 3 E. coli ST131-C2-6

$\triangle$ Exp. 3 E. coli ST131-A-14 (c)

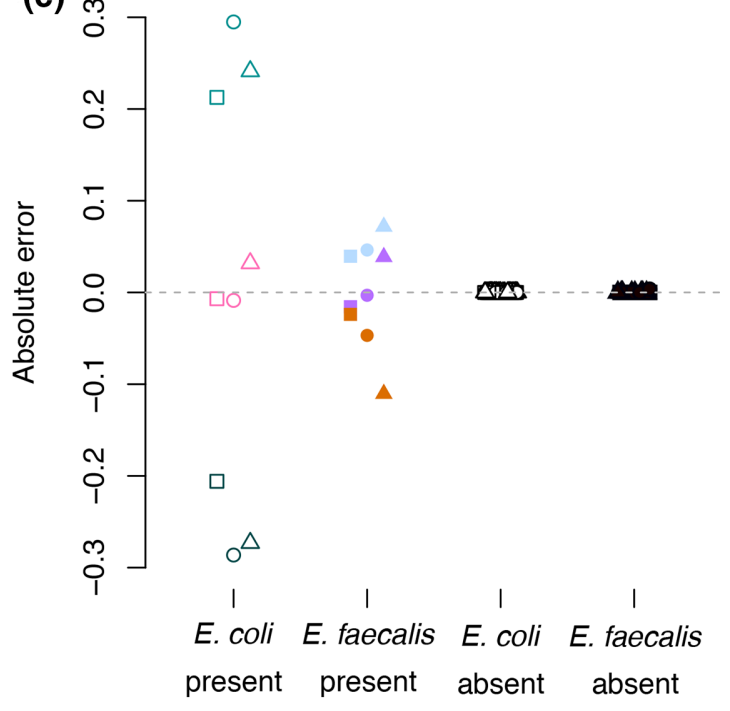

(d)

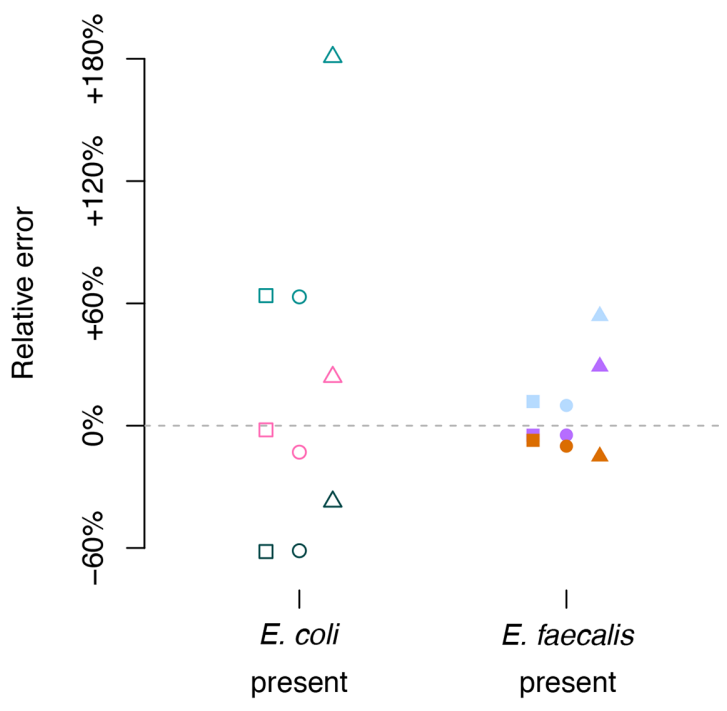

- Exp. 4 E. faecalis ST28

- Exp. 4 E. faecalis ST40

- Exp. 4 E. faecalis ST6

- Exp. 5 E. faecalis ST28

- Exp. 5 E. faecalis ST40

- Exp. 5 E. faecalis ST6

$\triangle$ Exp. 6 E. faecalis ST28

Exp. 6 E. faecalis ST40

$\triangle$ Exp. 6 E. faecalis ST6

Fig. 2. Evaluating mGEMS and mSWEEP on the in vitro benchmark data. Panels (a) E. coli and (b) E. faecalis compare the results of SNP calling from the isolate sequencing data (horizontal axis) against the results of SNP calling from the mixed samples with the mGEMS pipeline (vertical axis). The subplot in panel (b) contains a zoomed-in view of the points around the origin. Panels (c) and (d) compare the abundance estimates from mSWEEP to the ground truth relative abundances. Panel (c) shows the absolute difference between the estimates from mSWEEP and the true abundance. The values shown are split into E. coli and E. faecalis lineages truly present in the samples, and lineages truly absent. Panel (d) shows the relative error in the truly present lineages. 


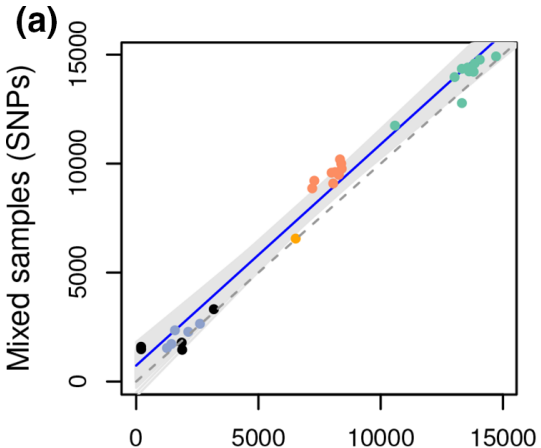

(d)

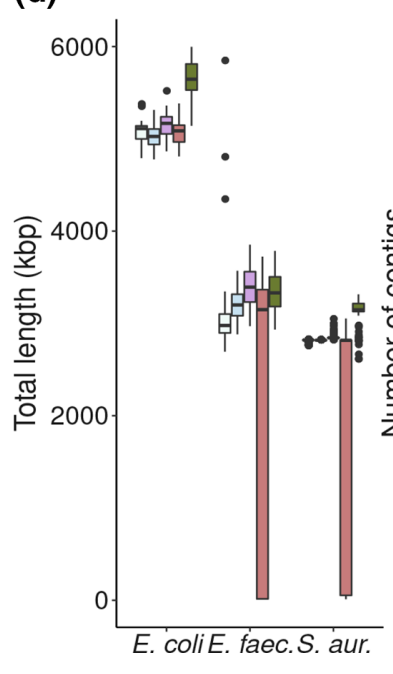

(b)

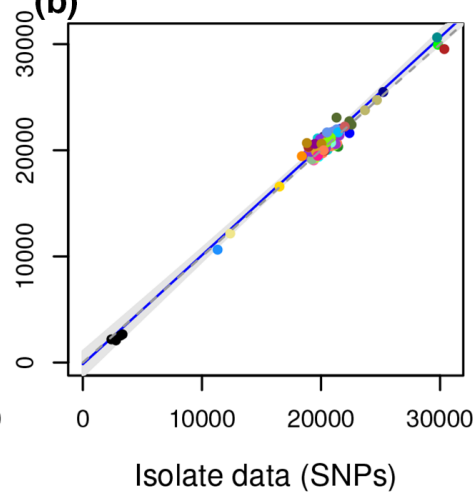

Isolate data (SNPS)

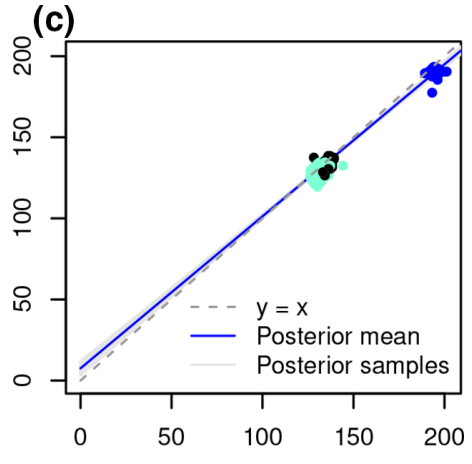

Fig. 3. Comparing mGEMS and synthetic mixtures with isolate sequencing data. Panels a-c compare the results of SNP calling from mixed samples with the mGEMS pipeline against the results from isolate sequencing data. Panel $d$ compares reference-free assembly statistics from mGEMS pipeline with different assemblers against the results from assembling the isolate sequencing data with Shovill. The results in panel a are for the E. coli ST131 isolates, panel b the E. faecalis isolates, and panel c the S. aureus ST22 isolates. In panels a and b, SNPs were called from contigs after assembling the reads. In panel c, the SNPs were called directly from the reads. Points are colored according to the lineage within the species (the full legend is available in Fig. S3). The dashed gray line represents a hypothetical perfect match between the binned and isolate reads. The blue line is the posterior mean while the shaded area contains the $95 \%$ posterior credible region calculated from 10000 posterior samples from a Bayesian regression model with the SNPs from the binned reads as the response and the SNPs from the isolate sequencing data as the sole explanatory variable. In panel d, the boxes are colored according to the type of assembly. The presented statistics are the summed lengths of all contigs (total length), the number of contigs, the sequence length of the shortest contig at $50 \%$ genome length (N50), and the smallest number of contigs whose sum of lengths is at least $50 \%$ of the genome length (L50).

suited to analysing the $S$. aureus samples since the number of SNPs between the strains is minimal (0-10 SNP differences within the lineages) and there are only three lineages, which translates poorly to finding a linear relationship.

\section{Split-k-mer comparison between isolate reads and mGEMS bins in synthetic mixtures}

We also examined the accuracy of the mGEMS binner without assembling by using the split $k$-mer analysis provided by the SKA software (v1.0, [64]). In a split- $k$-mer analysis, each nucleotide in the read is flanked by two $k$-mers. The nucleotide in the middle position plus the flanking $k$-mers constitute a single split- $k$-mer. If the split- $k$-mers are calculated for all nucleotides in two samples, they can be used to compare the samples on the basis of matching or mismatching split- $k$-mers or to call SNPs by comparing two split- $k$-mers where the flanking $k$-mers match but the nucleotide in between does not.

We first used SKA to call split-15-mer-SNPs in the three reference sequences from the binned sequencing reads, and calculated the difference in the count of SNPs called in the reference sequence between the isolate and the binned reads (Fig. S4). Since the results in Fig. 3 for S. aureus were obtained without assembly, there is no notable difference when compared to the SKA results. However, the SKA results for E. coli and E. faecalis contain fewer SNPs called from the binned reads, implying that binning with mGEMS acts as filtering for the sequencing data, since the results from the assemblies display no stark differences. 
In our next assessment, we performed pairwise comparisons within the separate sets of (1) all isolate reads, and (2) the binned reads. First we called the split-15-mer SNPs pairwise between all samples containing the isolate reads, and pairwise between all samples containing the binned reads. We then calculated the differences in the pairwise SNP counts obtained from the isolate reads and the binned reads. Secondly, we performed the same pairwise analysis but instead of the split-15-mer SNP counts we looked at the numbers of split15-mers that either were the same (matching) or different (mismatching) between each pair of samples. The results from these two comparisons (Fig. S5) show more discrepancy than the earlier results considering only SNPs called in the reference genome (Fig. 3), but the pairwise SNP counts are still relatively well preserved in all three species.

\section{Phylogenetic analysis of $E$. coli ST131 sublineages in a long-term care facility with synthetic mixtures}

We used a set of 30 multidrug-resistant E. coli ST131 strains sequenced from the residents of a long-term care facility in the UK [38] to produce a total of ten synthetic mixed samples. Each sample was the result of mixing isolate sequencing data from three E. coli ST131 sublineages (one from each of the main lineages $\mathrm{A}, \mathrm{B}$, or $\mathrm{C}$ ) together. We attempted to preserve the potential sequencing errors and biases by using all available reads from each of the isolate samples.

We applied the mGEMS pipeline to the ten synthetic mixed samples with a national (from the UK) collection of E. coli ST131 strains as the reference data [43], and used RAxML-NG (v0.8.1) [58] to infer a phylogenetic tree from both assemblies obtained from the isolate sequencing data (ground truth) and the assemblies from the mGEMS pipeline. Comparisons of these two trees (Fig. 4), show that the overall structure of the trees is highly similar, with the deep branches within the tree well reconstructed and differences in the tree topology appearing only at the very recent short branches.

\section{Population structure of nosocomial E. faecalis infections in the UK from synthetic mixtures}

Our next experiment was performed on sequencing data from bloodstream-infection-associated $E$. faecalis strains with a high prevalence of vancomycin resistance circulating in hospitals in the UK [39]. In this experiment, we mixed isolate sequencing data from seven distinct $E$. faecalis $S T$ Ts [45], producing a total of 12 synthetic mixed samples with seven lineages present in each. Each synthetic mixed sample included all sequencing reads from the mixed isolate sequencing data similarly to the $E$. coli experiment. We used a global collection of $E$. faecalis strains (all E. faecalis genome assemblies submitted to the NCBI as of 2 February 2020) as the reference data for the mGEMS pipeline, and again inferred the phylogenies for assemblies from both the isolate sequencing data and the results of the mGEMS pipeline. The more complex structure of these phylogenies was compared by plotting the two phylogenies against each other in a tanglegram (Fig. 5). Apart from a few structural mismatches in branches with poor bootstrap support values in both phylogenies (indicating uncertainty in the structure to begin with), the tree structure is strikingly well-recovered from the binned reads.

In fact, the tree inferred with the mGEMS pipeline has better bootstrap support values in the lower parts of the tree, suggesting that using mGEMS provides a better supported phylogeny than using the isolate sequencing data alone. We suspect this improvement in the bootstrap support values was caused by contamination in the isolate sequencing data for BSAC ec750, which produces an assembly 5.8 Mb long - nearly twice the length of the reference $E$. faecalis strain V583 (3.2 Mb). Similar changes in the bootstrap support values and additional structural changes occur in the parts of the tree containing the isolates BSAC ec294 and BSAC ec655 which both produce abnormally long assemblies (4.8 and $4.4 \mathrm{Mb}$, respectively). The assembly lengths for both the isolate and mGEMS-binned sequencing reads are provided in Table S1.

\section{Methicillin-resistant S. aureus transmission patterns among staff and patients at a veterinary hospital from synthetic mixtures}

In our last experiment, we used a dataset containing three $S$. aureus ST22 sublineages (called clade 1, clade 2, and clade 3) circulating among the staff and patients at a veterinary hospital in the UK [30] and separated by less than 150 SNPs. Because of the minimal differences between the clades, and a lack of isolates from these specific clades in published sources, we decided to use the isolates from the temporally first sample from the staff members as the reference data (representing a local reference collection). We separated the reference isolates from our experiment cases, which consist of all samples sequenced after the reference isolates, and proceeded to mix the remaining isolate sequencing data together. We generated a total of 312 synthetic mixed samples, each containing the sequencing data from three isolate samples from each of the three clades. Because the numbers of samples in each clade were not equal, the data from some of the isolate samples were included in multiple mixed samples. Since we wanted to represent each isolate with only a single instance in the phylogeny, we randomly chose one corresponding bin from mGEMS as the representative for an isolate that was included in multiple mixed samples.

The phylogenies in Figs. 6 and 7 were inferred with RAxMLNG (v0.8.1, [58]) from the results of the mGEMS pipeline. We plotted the subtrees of the overall phylogeny separately for the clade 1 isolates (Fig. 6) and clade 2 and 3 isolates (Fig. 7) without changing the underlying tree structure. Phylogenies inferred from the isolate sequencing data using the same pipeline are available in Figs S6 and S7. In the original study [30], staff member A was inferred as having introduced the MRSA strain from clade 1 into the veterinary hospital. In our phylogeny (Fig. 6), the initial samples from staff member A (timepoints labels 1 and 2) are indeed 
(a)
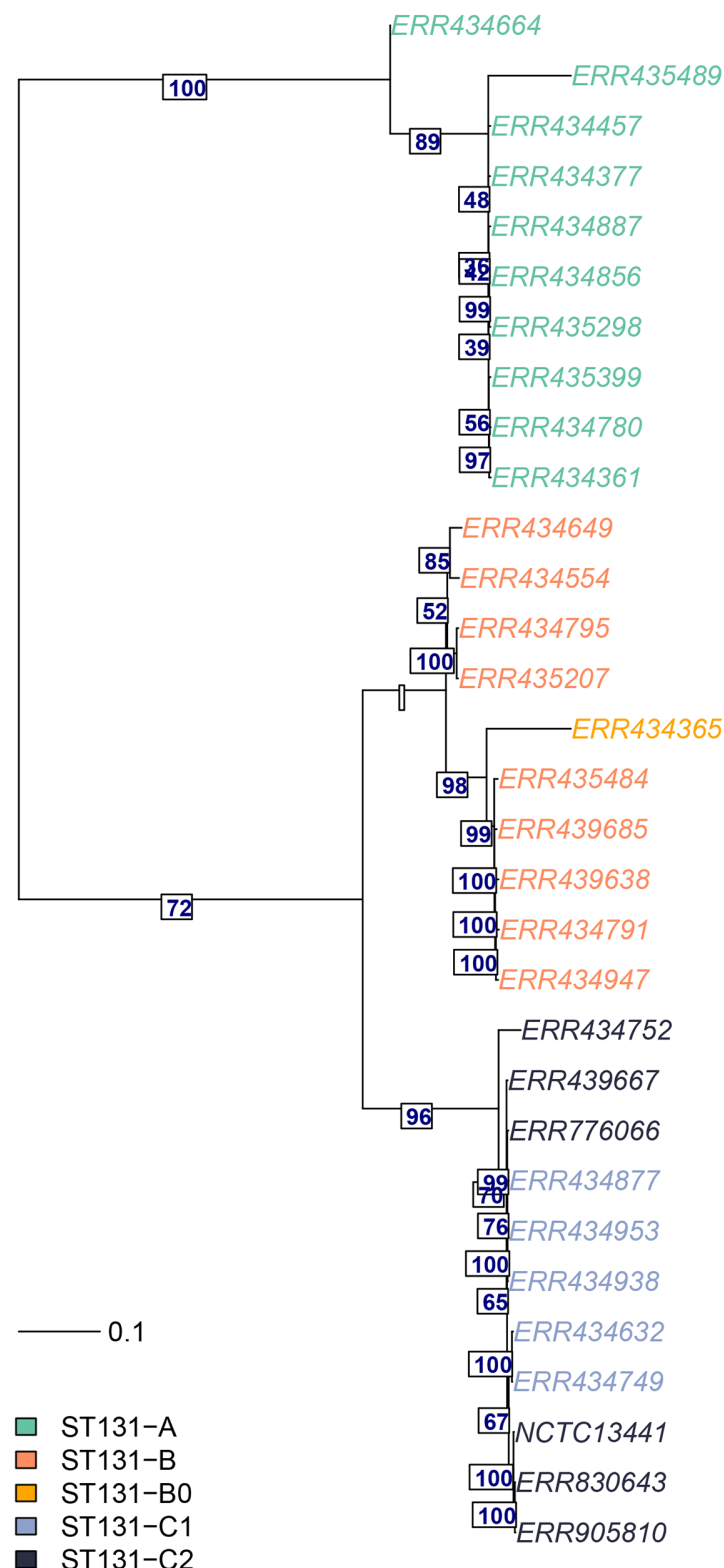

(b)

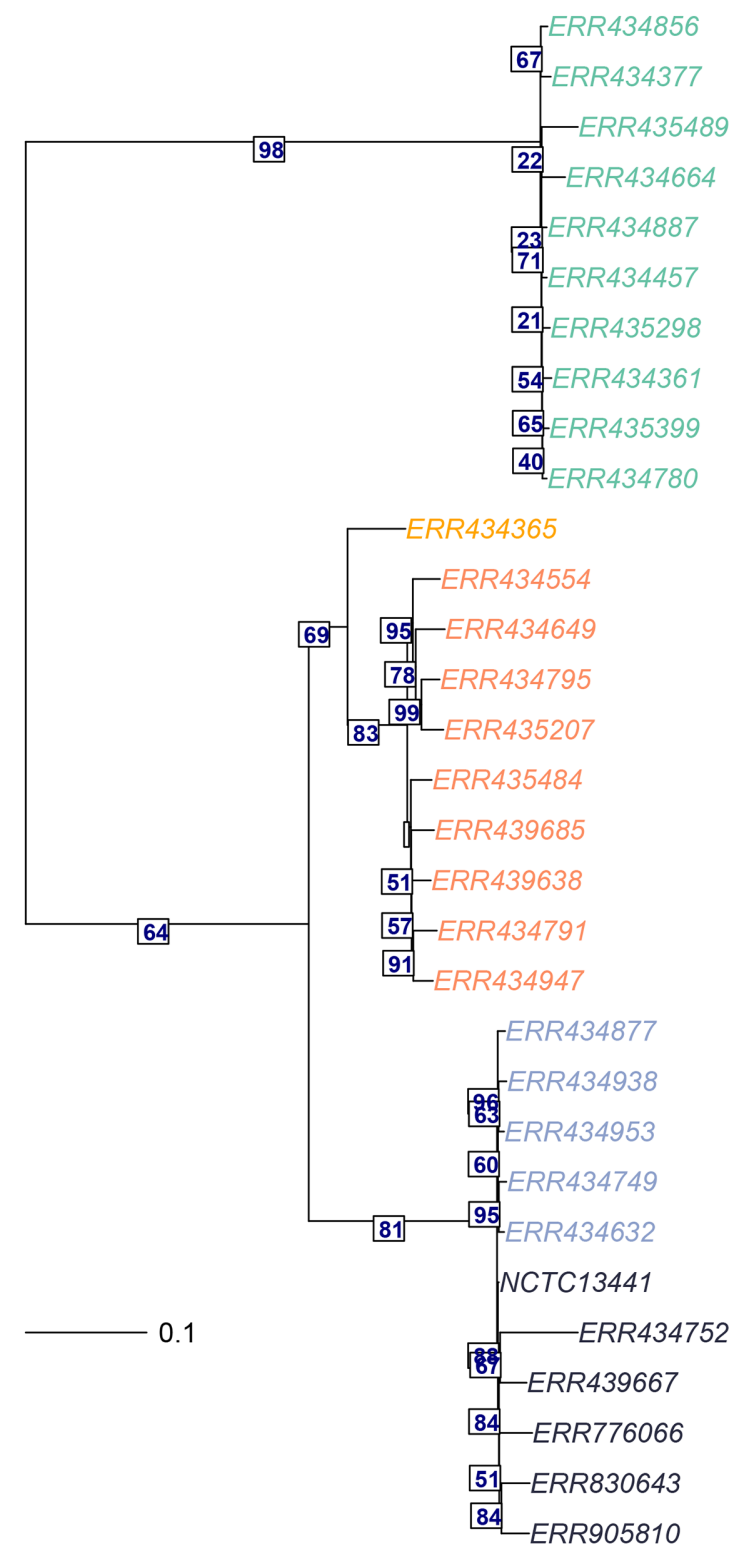

Fig. 4. Midpoint-rooted maximum likelihood trees from core SNP alignment of E. coli ST131 strains. The phylogeny in panel a was constructed from isolate sequencing data from 30 E. coli ST131 strains, and the phylogeny in panel b with the mGEMS pipeline from ten synthetic plate sweep samples, each mixing three isolate samples from the three main ST131 lineages (a-c; one strain from each per sample). Both phylogenies were inferred with RAxML-NG. Numbers below the edges are the branch support values from RAxML-NG for the next branch. Leaves are coloured according to the E. coli ST131 sublineage (a, b, B0, C1, or C2), and branch lengths in the tree scale with the mean number of nucleotide substitutions per site on the respective branch (GTR+G4 model). Leaves are labelled with the ENA accession number and the leaf labelled NCTC13411 corresponds to the reference strain used in calling the core SNPs.

contained at the root of the tree inferred from the mGEMS pipeline, although the placement of the strains further up the tree vary when compared to the results presented in the original study. The original study performed manual quality control of the SNP data by removing transposable elements which was not replicated in our experiment, possibly explaining some of the observed differences between the tree structures. The phylogenies for clades 2 and 3 (Fig. 7) follow the results of the original study more closely with most subclades found in both the isolate and the mixed sample phylogenies. Importantly, in all three clades no assembly from the mGEMS pipeline was assigned to the wrong clade in the phylogeny despite the minimal distances between the clades. 


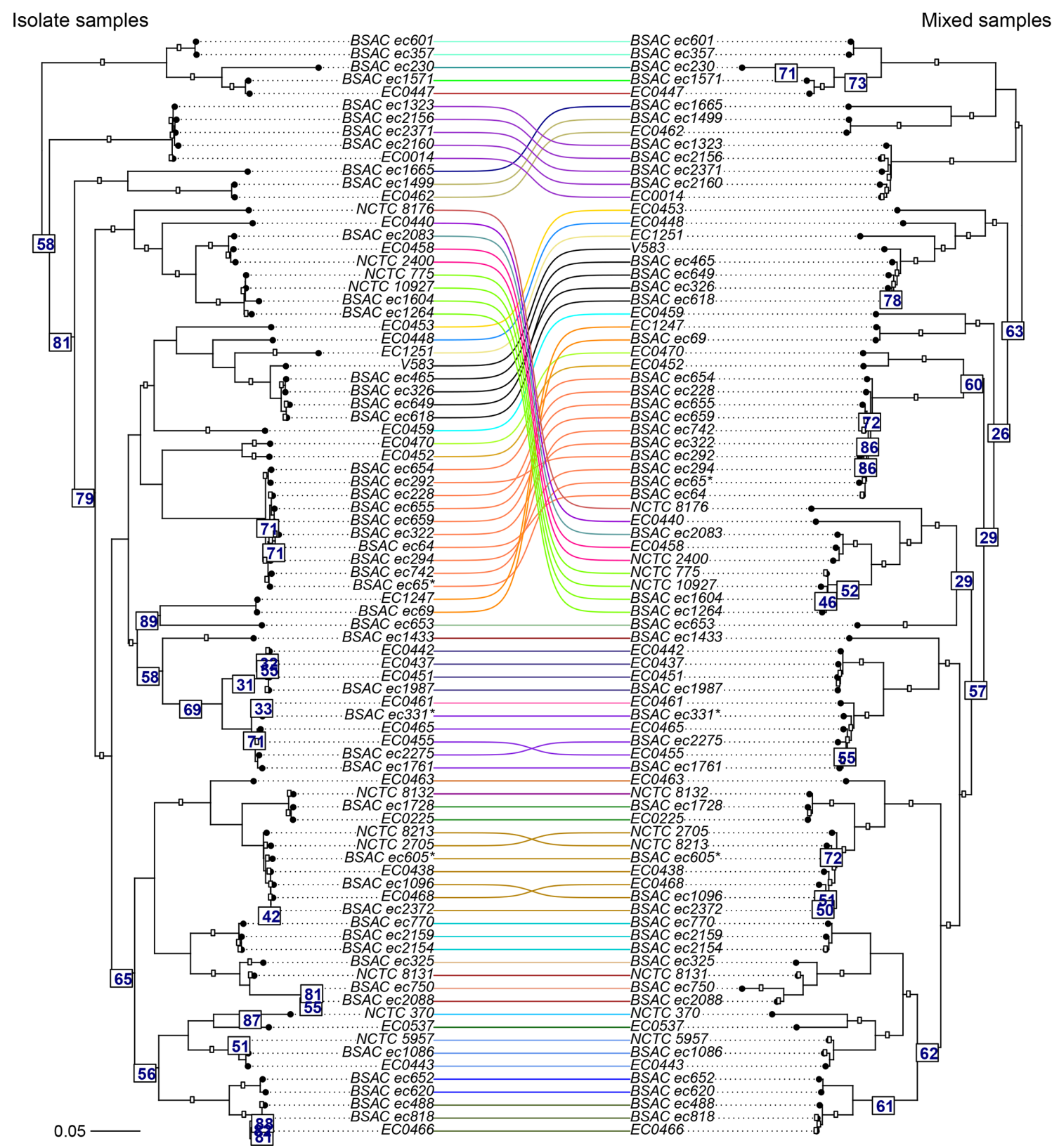

Fig. 5. Tanglegram of two midpoint-rooted maximum likelihood trees from core SNP alignment of E. faecalis strains. The phylogeny labelled Isolate samples (left side of the tree) was inferred with RAXML-NG from assembling the isolate sequencing data from 84 E. faecalis strains. The phylogeny labelled Mixed samples (right side of the tree) was inferred from 12 synthetic mixed samples, each containing sequencing data from seven different E. faecalis STs randomly chosen from the isolate sequencing data. Numbers below the edges indicate bootstrap support values from RAxML-NG for the next branch towards the leaves of the tree. Only support values less than 90 are shown. Branches are coloured according to the E. faecalis STs, and branch lengths in the tree scale with the mean number of nucleotide substitutions per site on the respective branch (GTR+G4 model). Leaves are labelled with the strain name from NCBI and the leaf labelled V583 corresponds to the reference strain for calling the core SNPs.

\section{DISCUSSION}

Adopting a plate-sweep approach, where DNA from the individual bacteria growing on the same plate is prepared and sequenced as a single library, shows clear promise in reducing the amount of manual and costly laboratory work that has been identified as an emerging bottleneck for epidemiological analyses at many public health laboratories [7]. We see two main applications for this. On the one hand, diagnostics where usually a single clone is picked can now capture the whole diversity at much lower additional costs. Whilst singleclone culturing might be required depending on the application (e.g. if more detailed speciation is required, or if further 


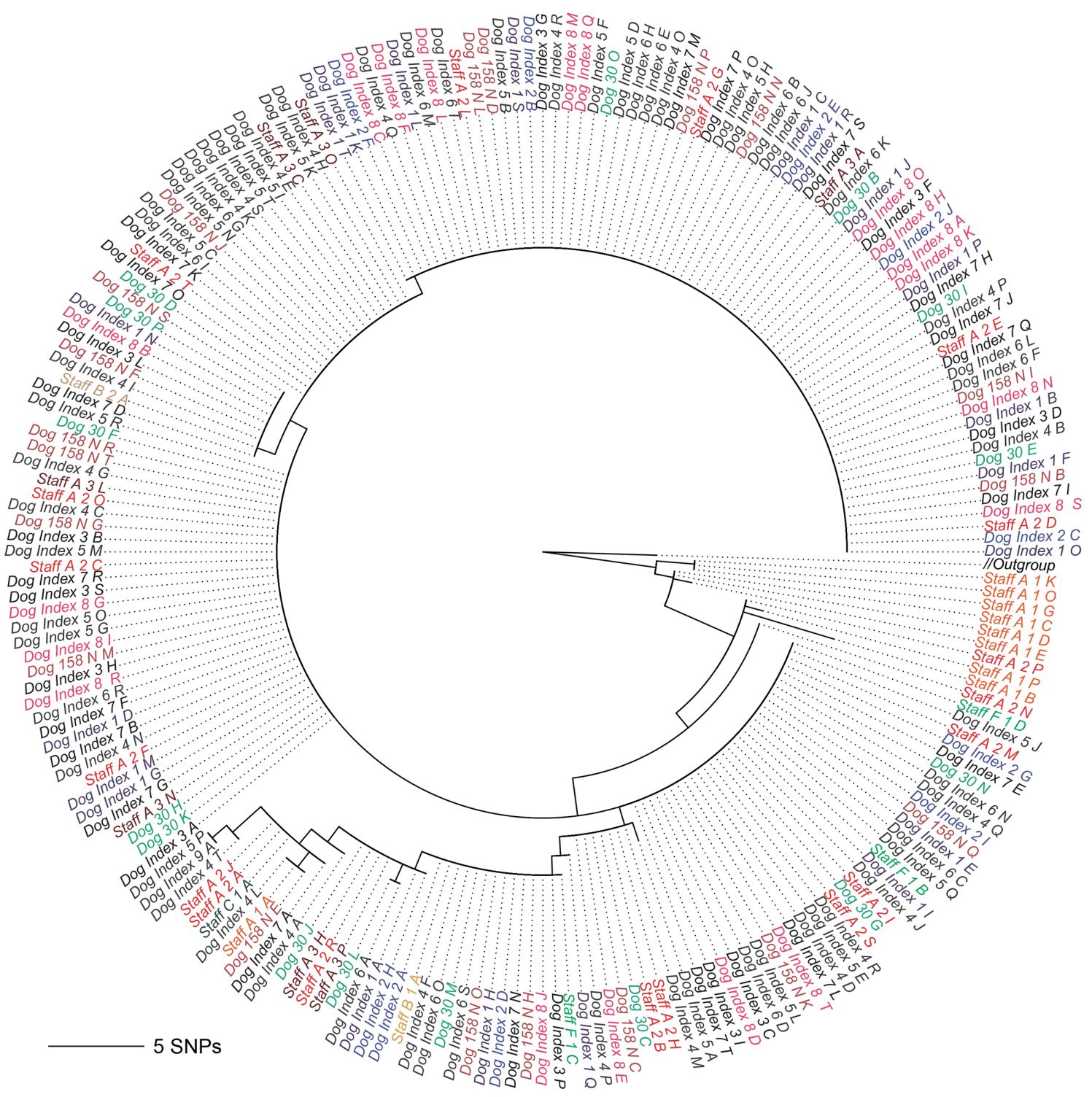

Fig. 6. Midpoint-rooted maximum likelihood tree from core SNP alignment of S. aureus ST22 showing strains from a single lineage within the sequence type. The phylogeny was inferred from a combined set of assemblies from 60 isolate sequencing samples (leaves labelled Staff A-G 1 A-T, corresponding to the temporally first samples from each staff member) and 312 assemblies obtained from the mGEMS pipeline applied to synthetic mixed samples of sequencing data from each of the three different S. aureus ST22 clades (1, 2, and 3). Only strains from clade 1 are displayed in the tree, with the branch labelled Outgroup leading to the collapsed clades 2 and 3. The mixed samples were produced from the isolate sequencing data collected from the patients, or from the staff members after the first sampling time. Branch labels are coloured according to the plate the isolate sequencing data was picked from. Branch lengths in the phylogeny scale with the mean number of SNPs obtained by multiplying the mean nucleotide substitutions per site on the respective branch (GTR+G4 model) with the total number of alignment sites. Leaves are labelled with the format: staff or patient, a letter indicating the donor, plate number (ascending in time), and a letter indicating the colony pick id.

phenotyping experiments are planned), a major cost- and time-factor - the DNA isolation, library prep and sequencing - are covered with a single experiment for the whole diversity, saving time and costs. On the other hand, this method might greatly support work on samples where the high diversity has so far proved as a major challenge, like longitudinal transmission or carriage studies especially in complex samples such as human faeces, where it is appreciated that only a fraction of the diversity can be covered with single-colony sequencing approaches.

In this article, we have introduced the mGEMS pipeline, which includes novel pseudoalignment and read binning methods, for genomic epidemiological analyses of plate sweeps. Our pipeline provides means to accurately recover the genomes, or corresponding sequencing reads, from mixed samples with 

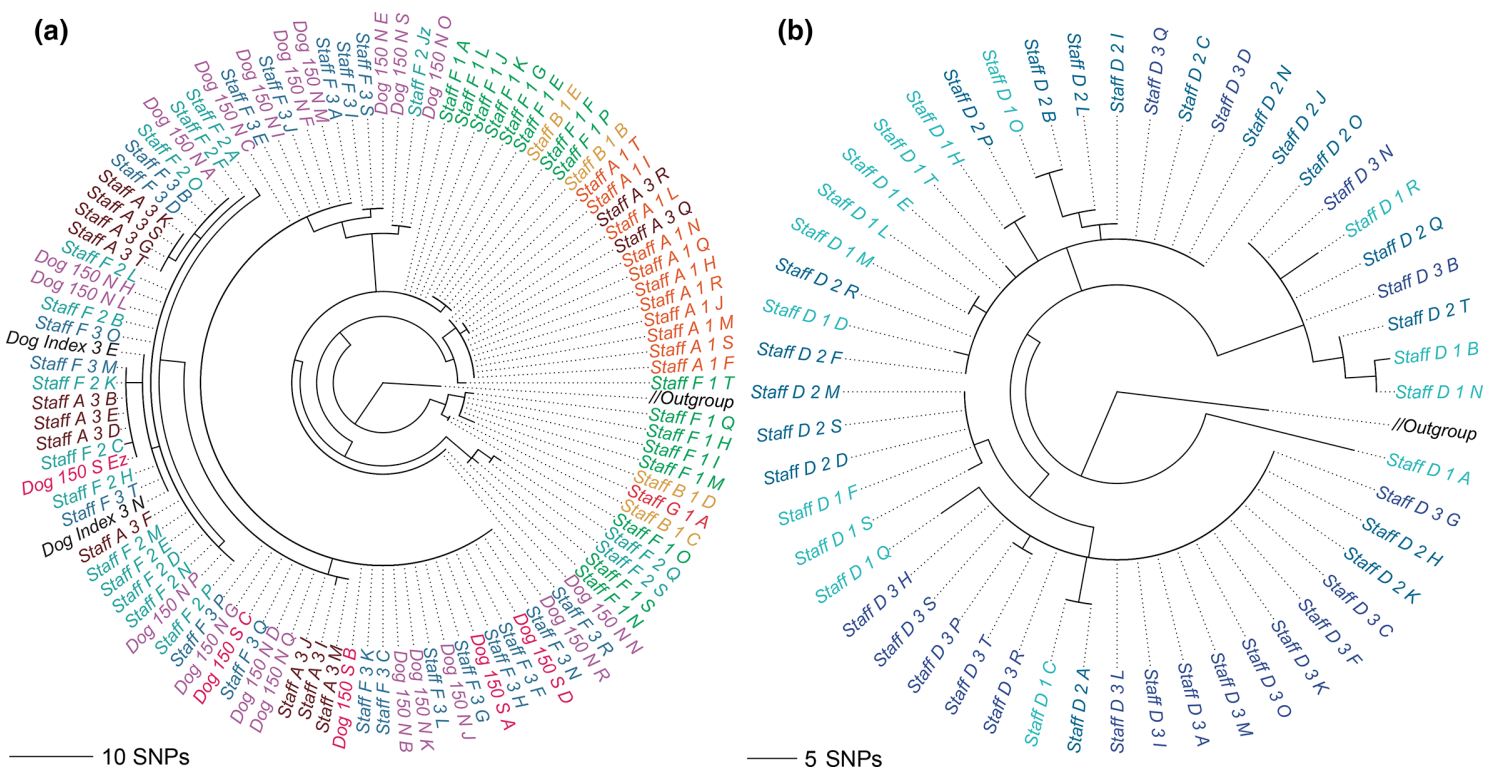

Fig. 7. Midpoint-rooted maximum likelihood trees from core SNP alignment of S. aureus ST22 showing clade 2 and clade 3 strains. The underlying phylogeny is the same as in Fig. 6. The phylogeny in panel a contains the clade 2 strains, and panel b the clade 3 strains. Branches leading to clade 1 and clade 3 (panel a), or clade 1 and clade 2 (panel b), labelled Outgroup in both panels, were collapsed. Branch labels are coloured according to the plate the isolate sequencing data was originally picked from with darker shades indicating later sampling times. Branch lengths in the phylogeny scale with the mean number of SNPs obtained by multiplying the mean nucleotide substitutions per site on the respective branch (GTR+G4 model) with the total number of alignment sites. Leaves are labelled with the format: staff or patient, a letter indicating the donor, plate number (ascending in time), and a letter indicating the colony pick id.

extremely closely related strains separated by less than a few dozen SNPs. In these settings, where the differences between the strains are at or under the sequence type level, isolate sequencing is traditionally required to draw epidemiological conclusions.

Using both samples based on synthetically mixed reads, as well as experimentally generated benchmark samples mixing bacterial DNA and strains, we have shown that with mGEMS we can robustly infer the same conclusions from plate sweeps that can be inferred from single-isolate sequencing data. Additionally, since mGEMS relies on modelling counts of pseudoalignments against grouped reference sequences, the inclusion of the alignment step causes the pipeline to also acts as quality control for sequencing reads from samples that inadvertently contain multiple lineages or contamination, which can disrupt downstream analyses like SNP calling [65]. In analysing sequencing data from closely related mixed samples, our pipeline reaches accuracy levels likely constrained by technical variation in the sequencing data and limitations in assembling sequencing data with variable coverage. Although existing tools like StrainPhlAn $[32,66]$ are capable of determining and analysing the dominant strains in complex mixed samples, to our knowledge mGEMS is the first tool capable of reliable recovery of the full strain variety when samples may contain multiple strains from the same species.
mGEMS demonstrates the power of plate sweep sequencing in genomic epidemiology and enables a change in the currently dominant framework that confers multiple benefits over both whole-genome shotgun metagenomics and isolate sequencing. Studies of the population structures of opportunistic pathogens have revealed extensive strain-level within-host variation $[21,23,27,67,68]$ with adverse implications for transmission analyses relying solely on isolate sequencing $[31,69]$ and colony pick based longitudinal studies reporting the absence or re-emergence of strains in a host $[30,38,70]$ or antimicrobial profiles $[28,71]$. While whole-genome shotgun metagenomics solves these issues to some extent $[35,72]$, the culture-free nature suffers from issues with both bacterial and host DNA contamination particularly affecting the sensitivity for detecting strains in low abundance $[29,33,34,73,74]$. Using mGEMS in conjunction with plate sweep sequencing data avoids these issues altogether, paving way for more representative studies of pathogen population structure and providing higher-resolution data for more complex models of transmission dynamics incorporating within-host variation and evolution [75-79].

mGEMS is designed for high-throughput short read sequencing data and requires a representative reference collection of the lineages present in the processed samples. When the reference data or the lineages are not sufficiently well-defined - like in the E. coli in vitro benchmark, where the unstable $E$. coli accessory genome was required 
to separate the two C2 strains into distinct lineages - the binning can be unreliable, leading to less accurate results. Mobile elements and plasmids that are not tied to a specific lineage may also pose a problem for mGEMS but we expect that their handling could be improved by including them as separate entities in the reference collection.

Since our method relies on available single-clone genomic reference data and plate cultures of the bacteria to sequence them at a sufficient depth for assembly, it obviously cannot be applied to the study of uncharacterized or unculturable species. However, culture media do exist for most human pathogens of public health relevance [37] or can be developed for some of the until recently unculturable bacteria [78-80]. Moreover, the availability of single-clone bacterial genome sequences is still increasing at a high rate, such that for many species or lineages plenty of sufficiently representative reference sequences would be available $[81,82]$. In these cases, the drastic reduction in the costs of library preparation, and the better capture of the underlying genomic variation between closely related bacteria in a set of mixed samples provided by mGEMS is extremely valuable. We hope that by enabling significant streamlining of the process of producing data for public health genomic epidemiology, our approach inspires both applications and further method development within this exciting research area.

\section{CONCLUSIONS}

We have developed the mGEMS pipeline for performing genomic epidemiological analyses from mixed samples containing multiple closely related bacterial strains. The two crucial novel enabling aspects introduced in this paper are the mGEMS read binner and the Themisto pseudoaligner. The mGEMS binner is a binning method based on turning probabilistic assignment of sequencing reads to reference lineages, while the Themisto pseudoaligner is a high-throughput exact pseudoaligner for short-read sequencing data that features external memory construction for compressed coloured de Bruijn graphs for scalability, providing significant runtime savings over conventional pseudoalignment. mGEMS addresses several major issues related to the cost, applicability, and sensitivity of the current approach in genomic epidemiology and enables entirely new types of analyses using mixed samples without sacrificing accuracy.

\section{Funding information}

TM and $\mathrm{AH}$ were supported by the Academy of Finland grant no. 310 261 as well as the Flagship programme (Finnish Centre for Artificial Intelligence FCAl; to JC and $\mathrm{AH}$ ). TK and JC were supported by the JPIAMR consortium SpARK (MR/R00241X/1). JC was funded by the ERC grant no. 742 158. TK was funded by the Norwegian Research Council JPIAMR grant no. 144501 . JA and VM were supported by the Academy of Finland grant no. 309048

\section{Acknowledgements}

The authors wish to acknowledge the Finnish Grid and Cloud Infrastructure (persistent identifier urn:nbn:fi:research-infras-2016072533), and CSC - IT Centre for Science, Finland for providing computational resources. Silje Lauksund for experimental assistance. Illumina sequencing for the in vitro mixture set-up and the Oxford Nanopore sequencing was performed at the Genomics Support Centre Tromsø, UiT The Arctic University of Norway.

\section{Author contributions}

T.M., T.K., J.C. and A.H., conceived the study, developed the full mGEMS pipeline, and designed the synthetic mixture benchmarking experiments. E.H., K.H., Ø.S., T.M., J.C. and A.H., designed the in vitro mixture study. K.H., Ø.S. and E.H., performed the experiments for the in vitro study. T.M. and A.H., developed the mGEMS binning algorithm. J.A. and V.M., developed the Themisto pseudoaligner. T.M., implemented the mGEMS binner. J.A., implemented the Themisto pseudoaligner. T.M., ran the experiments and created the visualisations. T.M., T.K., J.C. and A.H., interpreted the results and wrote the main article. JA wrote the supplementary file describing Themisto. K.H., Ø.S. and E.H., wrote the sections describing the in vitro mixture sample generation. All authors participated in reviewing and editing the article and discussed the results.

Conflicts of interest

The authors declare that there are no conflicts of interest.

Ethical statement

This study used only data from published sources, which have sought the appropriate ethical permissions.

\section{References}

1. Mäklin T, Kallonen T, Alanko J, Samuelsen $\emptyset$, Hegstad K, et al. Bacterial genomic epidemiology with mixed samples. Figshare, 2021.

2. Deng $X$, den Bakker HC, Hendriksen RS. Genomic epidemiology: whole-genome-sequencing-powered surveillance and outbreak investigation of foodborne bacterial pathogens. Annu Rev Food Sci Technol 2016;7:353-374.

3. Tang P, Croxen MA, Hasan MR, Hsiao WWL, Hoang LM. Infection control in the new age of genomic epidemiology. Am J Infect Control 2017:45:170-179.

4. Van Goethem N, Descamps T, Devleesschauwer B, Roosens NHC, Boon NAM, et al. Status and potential of bacterial genomics for public health practice: a scoping review. Implement Sci 2019;14:79.

5. Grad YH, Lipsitch M. Epidemiologic data and pathogen genome sequences: a powerful synergy for public health. Genome Biol 2014;15:538.

6. Kwong JC, Mccallum N, Sintchenko V, Howden BP. Whole genome sequencing in clinical and public health microbiology. Pathology 2015;47:199-210.

7. Rossen JWA, Friedrich AW, Moran-Gilad J. Practical issues in implementing whole-genome-sequencing in routine diagnostic microbiology. Clin Microbiol Infect 2018:24:355-360.

8. Scholz M, Ward DV, Pasolli E, Tolio T, Zolfo M, et al. Strain-level microbial epidemiology and population genomics from shotgun metagenomics. Nat Methods 2016;13:435-438.

9. Nurk S, Meleshko D, Korobeynikov A, Pevzner PA. metaSPAdes: a new versatile metagenomic assembler. Genome Res 2017:27:824-834

10. Li D, Luo R, Liu C-M, Leung C-M, Ting H-F, et al. MEGAHIT v1.0: A fast and scalable metagenome assembler driven by advanced methodologies and community practices. Methods 2016;102:3-11.

11. Li D, Liu C-M, Luo R, Sadakane K, Lam T-W. MEGAHIT: An ultrafast single-node solution for large and complex metagenomics assembly via succinct de BRUIJN graph. Bioinformatics 2015;31:1674-1676.

12. Peng Y, Leung HCM, Yiu SM, Chin FYL. IDBA-UD: a de novo assembler for single-cell and metagenomic sequencing data with highly uneven depth. Bioinformatics 2012;28:1420-1428.

13. Sieber CMK, Probst AJ, Sharrar A, Thomas BC, Hess M, et al. Recovery of genomes from metagenomes via a dereplication, aggregation and scoring strategy. Nat Microbiol 2018;3:836-843.

14. Kang DD, Li F, Kirton E, Thomas A, Egan R, et al. MetaBAT 2: an adaptive binning algorithm for robust and efficient genome reconstruction from metagenome assemblies. PeerJ 2019;7:e7359. 
15. Wu Y-W, Simmons BA, Singer SW. MaxBin 2.0: an automated binning algorithm to recover genomes from multiple metagenomic datasets. Bioinformatics 2016;32:605-607.

16. Breitwieser FP, Lu J, Salzberg SL. A review of methods and databases for metagenomic classification and assembly. Brief Bioinform 2019;20:1125-1136.

17. Sczyrba A, Hofmann P, Belmann P, Koslicki D, Janssen S, et al. Critical assessment of metagenome interpretation-a benchmark of metagenomics software. Nat Methods 2017;14:1063-1071.

18. McIntyre ABR, Ounit R, Afshinnekoo E, Prill RJ, Hénaff $E$, et al. Comprehensive benchmarking and ensemble approaches for metagenomic classifiers. Genome Biol 2017:18:182.

19. Vollmers J, Wiegand S, Kaster A-K. Comparing and evaluating metagenome assembly tools from a microbiologist's perspective - not only size matters! Rodriguez-Valera F, editor. PLOS ONE 2017;12:e0169662.

20. Meyer F, Hofmann P, Belmann P, Garrido-Oter R, Fritz A, et al. AMBER: Assessment of Metagenome BinnERs. Gigascience 2018;7:giy069.

21. Greenblum S, Carr R, Borenstein E. Extensive strain-level copynumber variation across human gut microbiome species. Cell 2015;160:583-594.

22. Ellegaard KM, Engel P. Beyond 16S rRNA community profiling: Intra-species diversity in the gut microbiota. Front Microbiol 2016:7:1475.

23. Schlager TA, Hendley JO, Bell AL, Whittam TS. Clonal diversity of Escherichia coli colonizing stools and urinary tracts of young girls. Infect Immun 2002;70:1225-1229.

24. Moreno E, Andreu A, Pérez T, Sabaté M, Johnson JR, et al. Relationship between Escherichia coli strains causing urinary tract infection in women and the dominant faecal flora of the same hosts. Epidemiol Infect 2006;134:1015-1023.

25. Lidin-Janson G, Kaijser B, Lincoln K, Olling S, Wedel H. The homogeneity of the faecal coliform flora of normal school-girls, characterized by serological and biochemical properties. Med Microbiol Immunol 1978;164:247-253.

26. Mosavie M, Blandy O, Jauneikaite E, Caldas I, Ellington MJ, et al. Sampling and diversity of Escherichia coli from the enteric microbiota in patients with Escherichia coli bacteraemia. BMC Res Notes 2019:12:335

27. Dixit OVA, O'Brien CL, Pavli P, Gordon DM. Within-host evolution versus immigration as a determinant of Escherichia coli diversity in the human gastrointestinal tract. Environ Microbiol 2018;20:993-1001.

28. Zlitni S, Bishara A, Moss EL, Tkachenko E, Kang JB, et al. Strainresolved microbiome sequencing reveals mobile elements that drive bacterial competition on a clinical timescale. Genome Med 2020;12:50

29. Kirkup BC. Bacterial strain diversity within wounds. Adv Wound Care (New Rochelle) 2015:4:12-23.

30. Paterson GK, Harrison EM, Murray GGR, Welch JJ, Warland JH, et al. Capturing the cloud of diversity reveals complexity and heterogeneity of MRSA carriage, infection and transmission. Nat Commun 2015;6:6560

31. Stoesser N, Sheppard AE, Moore CE, Golubchik T, Parry CM, et al. Extensive within-host diversity in fecally carried extendedspectrum-beta-lactamase-producing Escherichia coli isolates: implications for transmission analyses. J Clin Microbiol 2015;53:2122-2131.

32. Truong DT, Tett A, Pasolli E, Huttenhower C, Segata N. Microbial strain-level population structure and genetic diversity from metagenomes. Genome Res 2017;27:626-638.

33. Whelan FJ, Waddell B, Syed SA, Shekarriz S, Rabin HR, et al. Culture-enriched metagenomic sequencing enables in-depth profiling of the cystic fibrosis lung microbiota. Nat Microbiol 2020;5:379-390.
34. Ivy MI, Thoendel MJ, Jeraldo PR, Greenwood-Quaintance KE, Hanssen AD, et al. Direct detection and identification of prosthetic joint infection pathogens in synovial fluid by metagenomic shotgun sequenDetection and Identification of Prosthetic Joint Infection Pathogens in Synovial Fluid by Metagenomic Shotgun Sequencing. J Clin Microbiol 2018;56:00402-18

35. Gu W, Miller S, Chiu CY. Clinical metagenomic next-generation sequencing for pathogen detection. Annu Rev Pathol Mech Dis 2019:14:319-338.

36. Quince C, Walker AW, Simpson JT, Loman NJ, Segata N. Shotgun metagenomics, from sampling to analysis. Nat Biotechnol 2017:35:833-844.

37. Lagier J-C, Edouard S, Pagnier I, Mediannikov O, Drancourt M, et al. Current and past strategies for bacterial culture in clinical microbiology. Clin Microbiol Rev 2015;28:208-236.

38. Brodrick HJ, Raven KE, Kallonen T, Jamrozy D, Blane B, et al. Longitudinal genomic surveillance of multidrug-resistant Escherichia coli carriage in a long-term care facility in the United Kingdom. Genome Med 2017:9:70

39. Raven KE, Reuter S, Gouliouris T, Reynolds R, Russell JE, et al. Genome-based characterization of hospital-adapted Enterococcus faecalis lineages. Nat Microbiol 2016;1:15033.

40. Bray NL, Pimentel H, Melsted P, Pachter L. Near-optimal probabilistic RNA-seq quantification. Nat Biotechnol 2016:34:525-527.

41. Mäklin T, Kallonen T, David S, Boinett CJ, Pascoe B, et al. Highresolution sweep metagenomics using fast probabilistic inference. Wellcome Open Res 2020;5:14.

42. Seemann T. Shovill. 2018. https://github.com/tseemann/shovill

43. Kallonen T, Brodrick HJ, Harris SR, Corander J, Brown NM, et al Systematic longitudinal survey of invasive Escherichia coli in England demonstrates a stable population structure only transiently disturbed by the emergence of ST131. Genome Res 2017;27:1437-1449.

44. Lees JA, Harris SR, Tonkin-Hill G, Gladstone RA, SW L, et al. Fast and flexible bacterial genomic epidemiology with PopPUNK. Genome Res 2019:29:304-316.

45. Ruiz-Garbajosa P, Bonten MJM, Robinson DA, Top J, Nallapareddy SR et al. Multilocus sequence typing scheme for Enterococcus faecalis reveals hospital-adapted genetic complexes in a background of high rates of recombination. J Clin Microbiol 2006;44:2220-2228.

46. Jolley KA, Bray JE, Maiden MCJ. Open-access bacterial population genomics: BIGSdb software, the PubMLST.org website and their applications. Wellcome Open Res 2018;3:124.

47. Seemann T. mlst. GitHub, 2015. https://github.com/tseemann/ mlst

48. Paulsen IT, Banerjei L, Myers GSA, Nelson KE, Seshadri R, et al. Role of mobile DNA in the evolution of vancomycin-resistant Enterococcus faecalis. Science 2003;299:2071-2074.

49. Holden MTG, Hsu L-Y, Kurt K, Weinert LA, Mather AE, et al. A genomic portrait of the emergence, evolution, and global spread of a methicillin-resistant Staphylococcus aureus pandemic. Genome Res 2013;23:653-664.

50. Kolmogorov M, Yuan J, Lin Y, Pevzner PA. Assembly of long, errorprone reads using repeat graphs. Nat Biotechnol 2019;37:540-546.

51. Chen S, Zhou Y, Chen Y, Gu J. fastp: an ultra-fast all-in-one FASTQ preprocessor. Bioinformatics 2018:34:1884-90.

52. Walker BJ, Abeel T, Shea T. Priest M, Abouelliel A, et al. Pilon: An integrated tool for comprehensive microbial variant detection and genome assembly improvement. PloS one 2014:9:e112963.

53. Gagie T, Manzini G, Sirén J. Wheeler graphs: A framework for BWTbased data structures. Theor Comput Sci 2017;698:67-78.

54. Bankevich A, Nurk S, Antipov D, Gurevich AA, Dvorkin M, et al. SPAdes: a new genome assembly algorithm and its applications to single-cell sequencing. J Comput Biol 2012;19:455-477.

55. Nurk S, Bankevich A, Antipov D, Gurevich A, Korobeynikov A, et al. Assembling genomes and mini-metagenomes from highly chimeric reads. Deng $M$, Jiang R, Sun F and Zhang X (eds). In: 
Research in Computational Molecular Biology. Berlin, Heidelberg: Springer; 2013. pp. 158-170.

56. Seemann T. snippy: fast bacterial variant calling from NGS reads. GitHub, 2014. https://github.com/tseemann/snippy

57. Page AJ, Taylor B, Delaney AJ, Soares J, Seemann T, et al. SNPsites: Rapid efficient extraction of SNPs from multi-FASTA alignments. Microb Genomics 2016;2:e000056.

58. Kozlov AM, Darriba D, Flouri T, Morel B, Stamatakis A. RAxMLNG: a fast, scalable and user-friendly tool for maximum likelihood phylogenetic inference. Bioinformatics 2019:35:4453-4455.

59. Revell LJ. phytools: an R package for phylogenetic comparative biology (and other things. Methods Ecol Evol 2012;3:217-223.

60. Paradis E, Schliep K. ape 5.0: an environment for modern phylogenetics and evolutionary analyses in R. Bioinformatics 2019;35:526-528.

61. Bürkner P-C. Brms: An R package for bayesian multilevel models using stan. J Stat Softw 2017:80:1-28.

62. Bürkner P-C. Advanced bayesian multilevel modeling with the $R$ package brms. The R Journal 2018;10:395-411.

63. Carpenter B, Gelman A, Hoffman MD, Lee D, Goodrich B, et al. Stan: a probabilistic programming language. J Stat Softw 2017;76:1-32.

64. Harris SR. SKA: Split Kmer Analysis toolkit for bacterial genomic epidemiology. bioRxiv 2018;453142.

65. Goig GA, Blanco S, Garcia-Basteiro AL, Comas I. Contaminant DNA in bacterial sequencing experiments is a major source of false genetic variability. BMC Biol 2020;18:24.

66. Beghini F, Mclver LJ, Blanco-Miguez A, Dubois L, Asnicar F, et al. Integrating taxonomic, functional, and strain-level profiling of diverse microbial communities with bioBakery 3. eLife 2021;10:e65088.

67. Lieberman TD, Flett KB, Yelin I, Martin TR, McAdam AJ, et al. Genetic variation of a bacterial pathogen within individuals with cystic fibrosis provides a record of selective pressures. Nat Genet 2014:46:82-87.

68. Golubchik T, Batty EM, Miller RR, Farr H, Young BC, et al. Withinhost evolution of Staphylococcus aureus during asymptomatic carriage. PLoS One 2013;8:e61319.

69. Worby CJ, Lipsitch M, Hanage WP. Within-host bacterial diversity hinders accurate reconstruction of transmission networks from genomic distance data. PLoS Comput Biol 2014;10:e1003549.
70. Brodrick HJ, Raven KE, Harrison EM, Blane B, Reuter S, et al. Whole-genome sequencing reveals transmission of vancomycinresistant Enterococcus faecium in a healthcare network. Genome Med 2016;8:4.

71. Maciel JF, Gressler LT, Silveira BP, Dotto E, Balzan C, et al. Caution at choosing a particular colony-forming unit from faecal Escherichia coli: it may not represent the sample profile. Lett Appl Microbiol 2020;70:130-136.

72. Forbes JD, Knox NC, Ronholm J, Pagotto F, Reimer A. Metagenomics: the next culture-independent game changer. Front Microbiol 2017;8:1069.

73. McArdle AJ, Kaforou M. Sensitivity of shotgun metagenomics to host DNA: abundance estimates depend on bioinformatic tools and contamination is the main issue. Access Microbiol 2020;2:000104.

74. Salter SJ, Cox MJ, Turek EM, Calus ST, Cookson WO, et al. Reagent and laboratory contamination can critically impact sequencebased microbiome analyses. BMC Biol 2014;12:87.

75. De Maio N, Worby CJ, Wilson DJ, Stoesser N. Bayesian reconstruction of transmission within outbreaks using genomic variants. PLOS Comput Biol 2018;14:e1006117.

76. Worby CJ, Lipsitch M, Hanage WP. Shared genomic variants: identification of transmission routes using pathogen deep-sequence data. Am J Epidemiol 2017;186:1209-1216.

77. Skums P, Zelikovsky A, Singh R, Gussler W, Dimitrova Z, et al. QUENTIN: reconstruction of disease transmissions from viral quasispecies genomic data. Bioinformatics 2018;34:163-170.

78. Stewart EJ. Growing unculturable bacteria. J Bacteriol 2012;194:4151-4160.

79. Vartoukian SR, Palmer RM, Wade WG. Strategies for culture of 'unculturable' bacteria. FEMS Microbiol Lett 2010;309:1-7.

80. Ito T, Sekizuka T, Kishi N, Yamashita A, Kuroda M. Conventional culture methods with commercially available media unveil the presence of novel culturable bacteria. Gut Microbes 2019:10:77-91.

81. Forster SC, Kumar N, Anonye BO, Almeida A, Viciani E, et al. A human gut bacterial genome and culture collection for improved metagenomic analyses. Nat Biotechnol 2019;37:186-192.

82. Zou Y, Xue W, Luo G, Deng Z, Qin P, et al. 1,520 reference genomes from cultivated human gut bacteria enable functional microbiome analyses. Nat Biotechnol 2019:37:179-185.

\section{Five reasons to publish your next article with a Microbiology Society journal}

1. The Microbiology Society is a not-for-profit organization.

2. We offer fast and rigorous peer review - average time to first decision is 4-6 weeks.

3. Our journals have a global readership with subscriptions held in research institutions around the world.

4. $80 \%$ of our authors rate our submission process as 'excellent' or 'very good'

5. Your article will be published on an interactive journal platform with advanced metrics.

Find out more and submit your article at microbiologyresearch.org. 